This dissertation has been

$64-7698$

microfilmed exactly as received

ROSTRON, Robert Walther, 1937-

PRODUCTION OF METASTABLE Hg ATOMS

BY CHARGE EXCHANGE.

University of Arizona, Ph.D., 1964

Physics, electronics and electricity

University Microfilms, Inc., Ann Arbor, Michigan 


\section{PRODUCTION OF METASTABLE Hg ATOMS \\ BY CHARGE EXCHANGE}

by

Robert Walther Rostron

A Dissertation Submitted to the Faculty of the DEPARTMENT OF NUCLEAR ENGINEERING

In Partial Fulfillment of the Requirements For the Degree of

DOCTOR OF PHILOSOPHY

In the Graduate College

THE UNIVERSITY OF ARIZONA

1964 
THE UNIVERSITY OF ARIZONA

GRADUATE COLLEGE

I hereby recommend that this dissertation prepared under my direction by Robert Walthex Restron

entitled Production of Metastable Hg Atoms By Charge Exchange

be accepted as fulfilling the dissertation requirement of the degree of PheD.

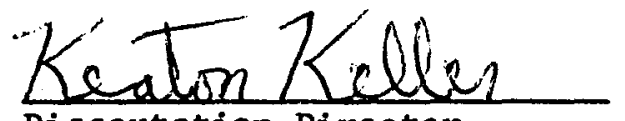

Dissertation Director
January 13,1964

Date

After inspection of the dissertation, the following members of the Final Examination Committee concur in its approval and recommend $i$ ts acceptance $* *$
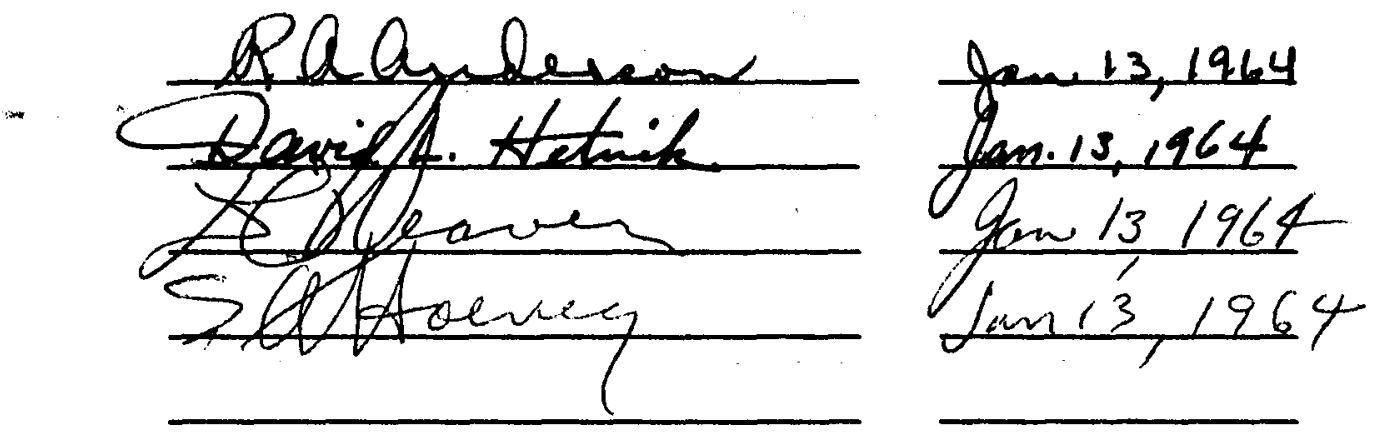

*This approval and acceptance is contingent on the candidate's adequate performance and defense of this dissertation at the final oral examination. The inclusion of this sheet bound into the library copy of the dissertation is evidence of satisfactory performance at the final examination. 


\section{STATEMENT BY AUTHOR}

This dissertation has been submitted in partial fulfillment of requirements for an advanced degree at The University of Arizona and is deposited in the University Library to be made available to borrowers under rules of the Library.

Brief quotations from this dissertation are allowable without special permission, provided that accurate acknowledgement of source is made. Requests for permission for extended quotation from or reproduction of this manuscript in whole or in part may be granted by the head of the major department or the Dean of the Graduate College when in their judgement the proposed use of the material is in the interests of scholarship. In all other instances, however, permission must be obtained from the author.

\section{SIGNED: Robert Walther Fostrov}

\section{APPROVAL BY DISSERTATION DIRECTORS}

This dissertation has been approved on the dates shown below:
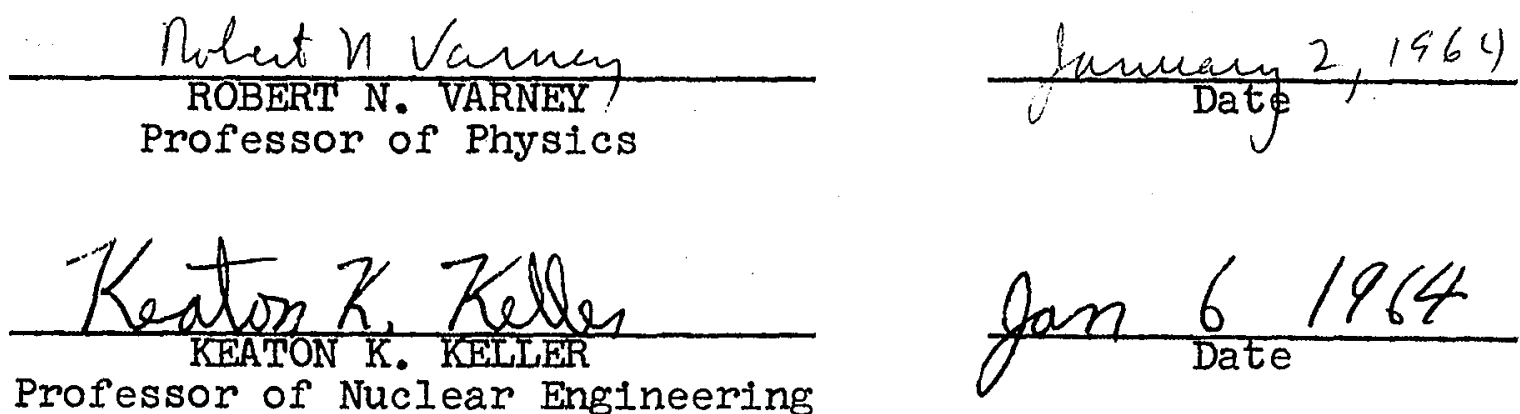


\section{$\underline{\text { ABSTRACT }}$}

Mercury Ions have been extracted from the plasma of a mercury arc through the small aperture in a probe at high negative potential. By means of sultable electrodes, the lons were brought to any desired energy in the range 100 to $4000 \mathrm{ev}$. The ions then passed through a field-free region containing mercury vapor where part of them became neutralized by charge-transfer collisions. The remaining ions were stopped by a retarding potential. The beam of neutral atoms then struck a metal target within a suitable cage for collecting ejected secondary electrons. The yield of secondary electrons was so large that it seemed unlikely that neutral atoms of mercury in their ground state could be responsible. Values of 8 as high as 0.40 were noted. The only reasonable alternative seemed to be that the neutral atoms were in a metastable state. Two methods were used to test this hypothesis. The first consisted of increasing the distance of flight of the metastables giving them more time to decay in flight. The second method was to introduce small amounts of foreign gases to quench the metastables in flight. In both cases the resulting large decreases in secondary electron current at the target confirmed the metastable hypothesis. These and other pertinent data are presented in the subsequent pages. 


\section{ACKNOWLEDGMENTS}

In the conduct of this research the writer is indebted for various degrees of aid from several persons and takes this opportunity to express appreciation for their help. The writer is particularly indebted to Dr. Keaton Keller of The University of Arizona Department of Nuclear Engineering for making possible the writing of this dissertation in absentia from the university.

The writer is also grateful to Mr. W. L. Sylvester and Mr. O. Retzloff of Washington University, St. Louis, Missouri, for their technical assistance in the construction of the experimental apparatus.

The author is deeply indebted to Dr. R. N. Varney of the Washington University Department of Physics for providing and directing the research problem undertaken as the subject of this dissertation. His constant encouragement and guldance proved invaluable in the successful completion of the research and dissertation.

The writer expresses his deepest gratitude to his loving wife and parents without whose faith and understanding this work could not have been completed. 


\section{Table of Contents}

CHAPTER 1 Introduction..................... I

CHAPTER 2 Theoretical Analysis.............. 8

2.1 Charge Transfer.................. 8

2.2 Secondary Emission by Excited Atoms........ 14

CHAPTER 3 Experimental Procedure.................. 17

3.I Experimental Apparatus.............. 17

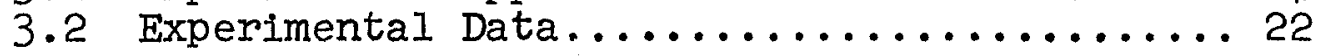

CHAPTER 4 Analysis of Results................. 31

4.1 Negative Ion Formation................... 31

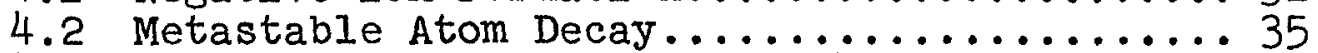

4.3 Quenching of Metastable Atoms by

Foreign Gases................... 40

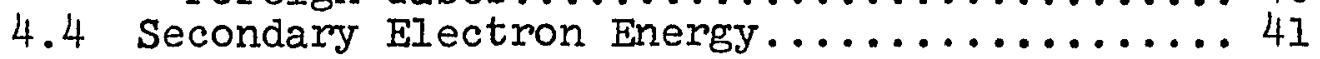

CHAPTER $5 . \quad$ Summary $\ldots \ldots \ldots \ldots \ldots \ldots \ldots \ldots \ldots$

BIBLIOGRA PHY ........................... 49 


\section{CHAPTER I}

\section{Introduction}

As the result of preliminary investigations by Varney $^{1}$ and Rostron ${ }^{2}$, it has been shown that fast neutral mercury atoms, formed by charge-transfer collisions, will produce a copious yield of secondary electrons from a metallic surface. In these studies a beam of positive mercury ions, which had been extracted from a mercury arc discharge, was allowed to pass through a field-free region containing mercury vapor. The ensuing charge-exchange processes provided a beam of fast neutral mercury atoms which were then directed against a metal target.

The resulting secondary emission was surmised to arise partially from intense local heating of the metallic surface due to the kinetic energy of the incident particles. However, based on the proposal of Varney that the chargetransfer process left the fast neutral mercury atoms in a

\section{$I_{R}$. N. Varney in private communication.}

${ }^{2}$. W. Rostron, "Secondary Emission Produced by Neutral Mercury Atoms", M.S. Thesis unpublished, University of Arizona, 1961. 
metastable ${ }^{3}$ state, the high yield was attributed mainly to a potential energy transfer between the impinging neutral. atoms and the electrons in the metal surface.

Earlier work supports the theory that metastable atoms will liberate electrons from a metallic surface by virtue of their potential energy. In $1924 \mathrm{Webb}^{4}$ demonstrated that thermal mercury atoms, excited by $4.86 \mathrm{ev}$ electron impacts, would eject electrons from a photo-sensitive surface. His procedure consisted of accelerating electrons from a heated cathode to $4.86 \mathrm{ev}$ and allowing these electrons to collide with mercury vapor atoms. The excited atoms formed in this manner would then diffuse to a photosensitive target. The potentials in the experimental tube were so arranged that no electrons could reach the target. Webb was perturbed by the fact that the Bohr theory did not predict the $4.86 \mathrm{ev}$ level in mercury to be metastable. He therefore believed that the excited neutrals would not have time to diffuse to the target before reverting to the ground state. In search of an explanation he postulated that it was the $4.86 \mathrm{ev}$ radiation, which he had observed, that was

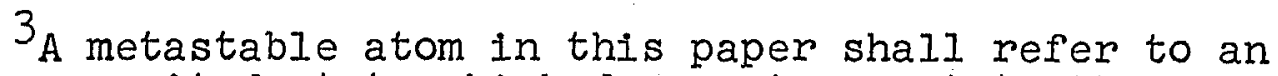
atom in an excited state which does not revert to the ground state by a radiative transition i.e., Its lifetime is of the order of $10^{-7} \mathrm{sec}$. or greater.

${ }^{4} \mathrm{H}$. W. Webb, "The Metastable State in Mercury Vapor", Physical Review, Vol. 24, (1924), p. 113. 
responsible for the photo electrons.

In later work by Webb and Messenger ${ }^{5}$, the photo-sensitive target was shielded from the source of excited mercury atoms by means of quartz and calcite crystal. Such a shield would prevent the excited atoms from reaching the target but would transmit the radiation. In this manner it was demonstrated conclusively that it was indeed the excited atoms which were responsible for the electron ejection from the target. The question of the ilfetime of the $4.86 \mathrm{ev}$ state remained unanswered.

Webb's dilemma concerning the stability of the 4.86 ev $\left(3_{\mathrm{P}_{1}}\right)$ state has since been resolved by the establishment of the $4.66 \mathrm{ev}\left(3_{\mathrm{P}_{0}}\right)$ and $5.43 \mathrm{ev}\left(3_{\mathrm{P}_{2}}\right)$ metastable states in mercury. As late as 1958 Lichten $^{6}$ further established a 9.0 ev metastable state by means of secondary emission studies. He believed this to be the ${ }^{3} \mathrm{D}_{3}$ state and predicted its lifetime to be of the order of 0.01 seconds. Thus it is likely that Webb actually observed the secondary emission effect of the $4.66 \mathrm{ev}$ metastables and the radiation from the $4.86 \mathrm{ev}$ state simultaneously.

5H. A. Messenger, "The Significance of Certain critical Potentials of Mercury in Terms of Metastable Atoms and Radiation", Physical Review, Vol. 28, (1926) p. 962.

6W. Lichten, "New Metastable State of Mercury", Physical Review, Vol. 109, (1958), p. 1191. 
Coulliette ${ }^{7}$ in 1928 studied the diffusion of metastable mercury atoms in mercury vapor. In addition to verifying the work of Webb and Messenger, he succeeded in showIng that the collision radius of the metastable atom is about 1.5 times that of the ground state atom. In addition he found evidence that the metastable atoms lost excitation energy as a result of collisions with ground state atoms.

In 1933 Sonkin $^{8}$ studied the action of slow mercury metastables on metallic targets subjected to various environmental conditions. His investigations indicated that a deposit of mercury on the target was essential to a surface sensitive to metastables. Atomically clean targets, produced by heating the metal, were found to be comparatively poor secondary electron emitters.

The first efforts to study secondary emission by fast neutral mercury atoms were undertaken by Chaudhri and Kahn 9 in 1948. They succeeded in bombarding a target with a beam

7 J. H. Coulliette, "The Diffusion of Metastable Atoms in Mercury Vapor", Physical Review, Vol. 32, (1928), p. 636 .

${ }^{8}$ S. Sonkin, "The Action of Mercury Metastable Atoms on a Tungsten Surface", Physical Review, Vol. 43, (1933), p. 788 .

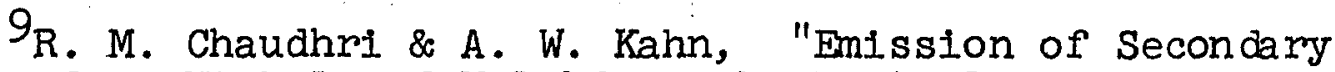
Electrons from Nickel and Molybdenum by Neutral Atoms of Mercury and Potassium", Proceedings of the Physical Society of Iondon, Vol. 61, (1948), p. 526. 
of fast neutrals ( $0-5500 \mathrm{ev}$ ) produced by the grazing Incidences of positive mercury ions with the walls of a nickel canal. The ions, formed by electron impacts with mercury vapor atoms, were extracted from the vapor and accelerated to high speeds by means of a cylindrical probe electrode. The probe doubled as the neutralizing canal, and the neutrals formed by grazing incidences of the ions with the canal walls continued to the target without suffering appreciable deflections. The secondary electron energy spectrum from the target was observed by Chaudhri and Kahn to be Maxwellian. As a result they concluded that the ejection of electrons was due only to the local heating induced at the metallic surface by the kinetic energy of the neutrals.

Experiments closely paralleling those of Chaudhri and Kahn were carried out independently by Oliphant ${ }^{10}$ (1929), Berry $^{11}$ (1948), and Hagstrum ${ }^{12}$ (1954) with ions and neutral atoms of the noble gases. Their studies revealed that the yield of secondary electrons was nearly independent of the

10M. L. E. Oliphant, "The Action of Metastable Atoms of Helium on a Metal Surface", Proceedings of the Royal Soclety, Vol. Al24, (1929), p. 228.

${ }^{11_{\mathrm{H}}}$. W. Berry, "Secondary Electron Emission by Fast Neutral Molecules and Neutralization of Positive Ions", Physical Review, Vol. 74, (1948), p. 848.

\section{$12 \mathrm{H}$. Hagstrum, "Auger Ejection of Electrons From} Tungsten by Noble Gas Ions", Physical Review, Vol.96, (1954), p. 336 . 
kinetic energy of the incident particles over the energy range $0-4000 \mathrm{ev}$. The yields were also found to be independent of nuclear mass. These results substantiated the theoretical predictions of Cabas and Lamb ${ }^{13}$ (1944) and Hagstrum ${ }^{14}$ (1954) that secondary emission by lons and atoms is essentialiy a potential energy transfer phenomenon.

The research undertaken as the subject of this dissertation has been an attempt to validate the theory that metastable mercury atoms are formed in charge transfer collisions. The procedure consisted of bombarding a metallic target with neutral mercury atoms formed by charge exchange collisions. The intensity of the ensuing secondary electron current from the target was then studied as a function of the target distance from the charge-exchange region. An exponential decrease of secondary electron current, with increasing target distance, would indicate that the neutrals were in an excited quantum state and decayed to the ground state in flight. A second method of quenching the presumed metastable atoms consisted of admitting small amounts of inert gases into the region between the charge-exchange

13 A. Cobas \& W. Lamb, "On the Extraction of Electrons from a Metal Surface by Ions and Metastable Atoms", Physical Review, Vol. 65, (1944); p. 327 .

$14 \mathrm{H}$. Hagstmum, "Theory of Auger Ejection of Electrons From Metals By Ions", Physical Review, Vol. 96, (1954), p. 336 . 
region and the target. The results of these operations as well as other pertinent data will be presented in subsequent chapters. 
CHAPTER 2

Theoretical Analysis

Two basic phenomena are of interest in the analysis of this experiment. The first entails the charge-transfer process between a positive ion and a neutral atom, while the second is the secondary emission produced by neutral atoms. The mercury arc discharge ${ }^{l}$, which acts as the source of mercury ions in the experiment, also lends itself readily to analysis. However, since the subject of mercury discharges is thoroughly covered in the literature ${ }^{2}$, it will not be treated here.

\section{I Charge Transfer}

The passage of ions through their own gas, or another species of gas, is subject to the phenomenon of chargeexchange between the ion and the neutral gas atom. Several of the possible charge-transfer processes are listed below:

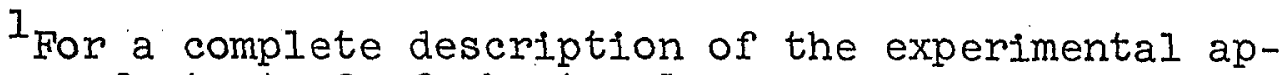
paratus see footnote 2 of chapter 1 .

${ }^{2}$ See for example Fundamental Processes of Electrical Discharge in Gases, by L. B. Loeb. 
1. Single charge transfer..... $A^{+}+B \rightarrow A+B^{+}$

2. Transfer Ionization......... $A^{+}+B \rightarrow A+B^{2+}+e$

3. Stripping.............. $A^{+}+B \rightarrow A^{2+}+B+e$

4. Charge transfer to an excited level............. $A^{+}+B \rightarrow A^{*}+B^{+}$

5. Capture by neutral atom.... A $+B \rightarrow A^{-}+B^{+}$

6. Collisional detachment...... $A^{-}+B \rightarrow A+B+e$ The single-charge-transfer process has been more thoroughly studied than the competing inelastic collision processes. This may be attributed to the predominating probability of the process. Single charge transfer results in the formation of a very slow ion $\left(B^{+}\right)$, and an atom (A) which has nearly the same kinetic energy as the primary ion $\left(A^{+}\right)$. Thus upon traveling one charge-transfer mean free path, the primary ion $\left(\mathrm{A}^{+}\right)$becomes a neutral atom but retains its velocity. The atom (B), which has lost an electron to the ion and has itself become an ion, begins to drift with thermal velocity.

The effective collision cross section between two atoms in which electronic transitions occur goes through a maximum when the difference in internal energy $\Delta E$ is a minimum. The collision is considered to be a gradual phenomenon, where the atoms approach each other with a velocity which is small compared to that of the atomic electrons. This may be represented by considering the amplitude of the 
vibration set up when a disturbing force is applied to an oscillator of natural frequency $D$. This disturbance will vary with time according to some function of the force. If this function is expanded in a Fourler integral, only the components with frequencies between $P$ and $P+d P$ will produce any appreclable forced oscillations. In order for the components of the force to be large, the time of collision $\tau$ should be of the order of the natural period of the oscillator. Taking $\boldsymbol{\tau}$ to be of the order $a / v$, where " $a$ " is the range of interaction between the atoms and " $v$ " is their relative velocity, the condition for maximum excitation is $(\operatorname{ap} / v)=1$. To transfer this classical picture to quantum theory it is only necessary to replace $P$ by $\Delta E / h$. The transition probability is therefore a maximum if $(a \Delta E / h v)=1.3$

Measurements of the charge-transfer-collision cross sections are consistent with this picture. The cross section for an ion traveling through its own gas is greatest when its kinetic energy is least as illustrated in figure 2.11. For ions passing through a different vehicular gas, the cross section passes through a maximum. This maximum shifts to higher velocities as the difference in ionization

${ }^{3}$ S. C. Brown, Basic Data of Plasma Physics, Wlley \& Sons, New York, 1959, $\frac{\text { pp. } 36-40 .}{6}$ 


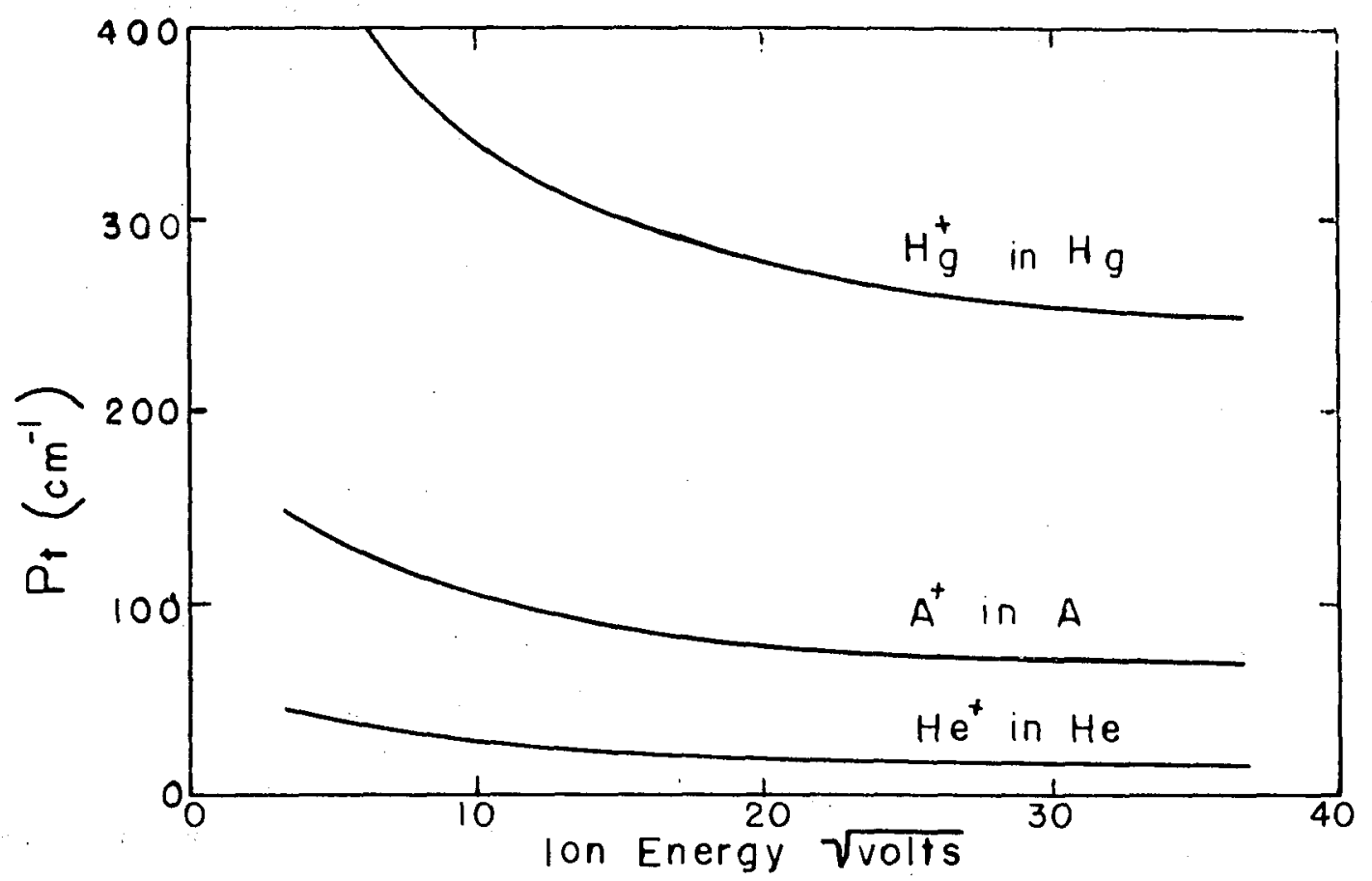

FIG. 2.11 Resonance Chorge-Tronsfer Cross Sections

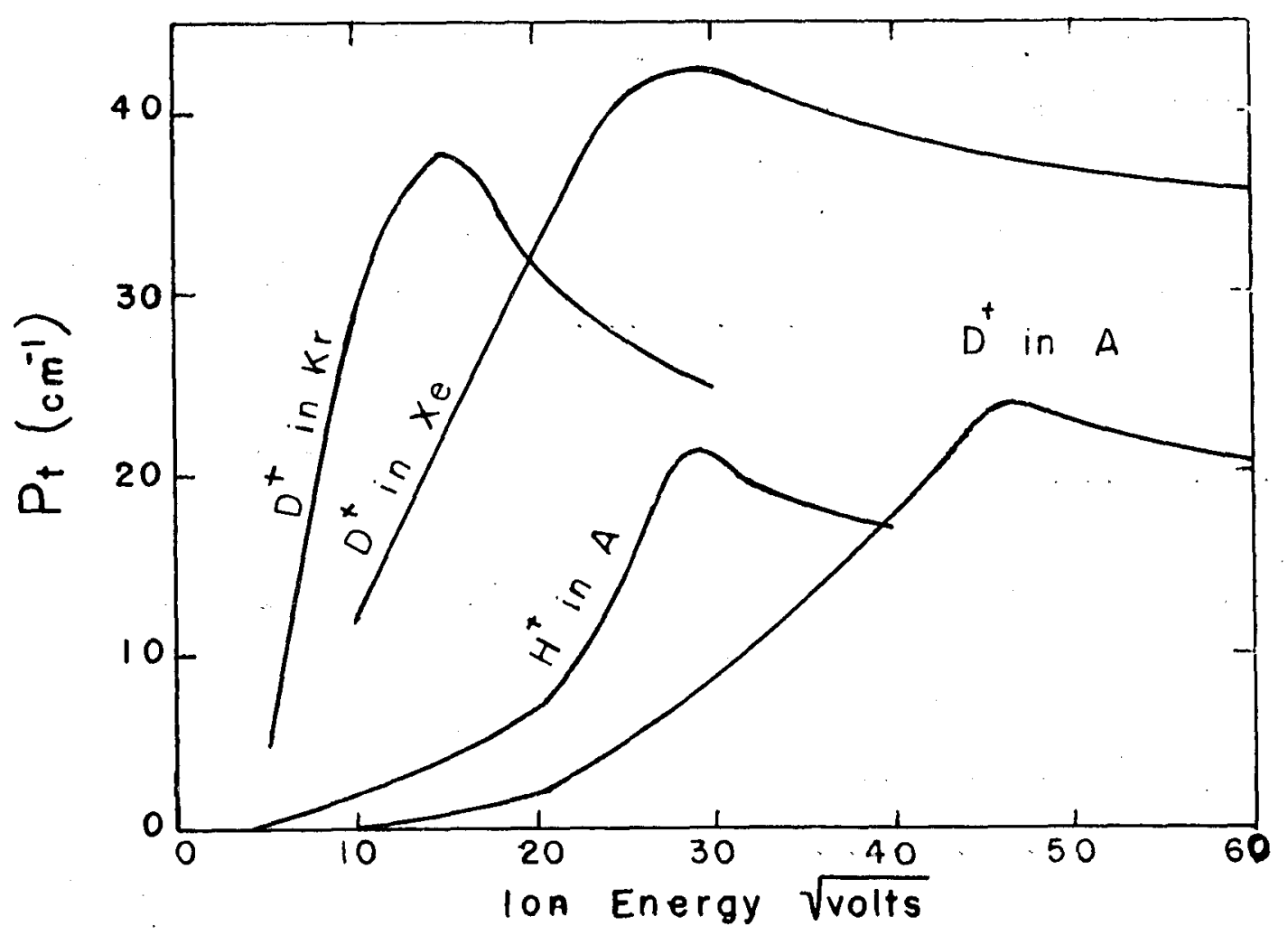

FIG.2.12 Non-Resonance Charge-Transfer Cross Sections 
potentials increases as depicted in figure 2.12. Because of the very large cross sections involved in single charge transfer between positive ions and atoms of the same.specles, this process has become known as symmetrical resonance charge transfer. 4

When the difference in velocities between the ion and the atom of the same gas becomes large, the chargetransfer cross section asymptotically approaches a constant value that depends upon the Ionization potential of the atom. Sena ${ }^{5}$ has worked out an approximate theory for this, which gives the limiting macroscopic cross section as:

$$
P_{t}=\frac{1.88 \times 10^{4}}{u} \mathrm{~cm}^{-1} \quad \begin{aligned}
& \text { at } 1 \mathrm{~mm} \text { pressure } \\
& \text { and } 0^{\circ} \mathrm{C} .
\end{aligned}
$$

where " $u$ " is the ionization potential.

A general theory applicable to any charge-exchange collision between an atomic ion and an atom has been worked out by Holstein ${ }^{6}$. He has shown that the region in which the charge-transfer collision will occur may be considered a sphere of radius $R_{c}$ surrounding the atom. Outside $R_{c}$ the

${ }^{4}$ s. C. Brown, op. cit., pp. 40-41.

5L. Sena, J. Exp. Theoretical Phys. (U.S.S.R.) 9, 1320, (1939).

6. Holstein, J. Phys. Chem. 56, 832 (1952). 
probability of charge-transfer falls rapidly to zero, while Inside $R_{c}$ it is $\frac{1}{2}$. As an ion approaches an atom, it will be deflected toward the atom as a result of the dipole moment it induces in the atom. Since the radius $R_{c}$ for chargetransfer in resonance cases is very large, an ion passing outside $R_{c}$ is almost undeflected by the dipole moment forces. In the non-resonance cases, $R_{c}$ is so much smaller that attractive polarization forces are responsible for scattering of ions passing near to atoms but outside $R_{c}$. Thus if the ion enters the charge-transfer sphere in either case, it emerges half the time as a neutral atom and the other half of the time remains an ion. According to this model, the probability of charge-transfer oscillates rapidly between zero and unity for impact parameters equal to or less than $R_{c}$. The mean probability in this range is $\frac{1}{2}$ so that the microscopic charge-transfer cross section is given by $\sigma=\frac{1}{2} \pi R_{c}^{2}$.

The charge-transfer process predicted in this experiment may be represented by the following expression:

$$
\text { (Eq. 2.11) } \mathrm{Hg}^{+}+\mathrm{Hg} \rightarrow \mathrm{Hg}^{*}+\mathrm{Hg}^{+}
$$

This process is a single-charge-transfer phenomenon between two similar atomic species in which one of the products is in an excited state. The discovery of the excited state of the neutralized mercury ion is one of the features of the 
present work. As a result of the formation of the excited mercury atom, the process of equation 2.11 cannot rigorously be defined as symmetrical resonance charge-transfer. However, from the high yield of metastable atoms observed, as well as from the fact that both reactants are mercury, the reaction appears to be a resonance process. This is further substantiated by the behavior of the charge-transfer cross section for mercury ions in mercury vapor as illustrated in figure 2.11. The dilemma may be resolved by assuming that the incident mercury ion is itself in an excited state and transfers this excitation energy to the neutral atom. In this manner potential energy is conserved in the collision. The equation for this process is given in equation 2.12 .

$$
\text { (Eq. 2.12) } \mathrm{Hg}^{+*}+\mathrm{Hg} \rightarrow \mathrm{Hg}^{*}+\mathrm{Hg}^{+}
$$

The assumption that metastable ions exist is substantiated by the work of Hagstrum ${ }^{7}$ who studied extensively several species of excited ions.

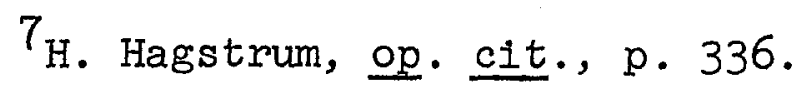


2.2 Secondary Emission by Excited Atoms

When a neutral atom in an exclted quantum state enters the vicinity of a metalic surface, it may lose its excitation energy with the resulting ejection of an electron from the metal. Early theory predicted that during such a collision of an excited atom with a metallic surface, the excited electron simply dropped to the ground state with the emission of a quantum of radiation. This radiation was in turn supposed to eject an electron from the metal by a photo-electric process.

Application of quantum mechanics to the subject of secondary emission has led to a more sophisticated picture of this process. Consider the energy level diagram of the metallic surface and excited atom shown in figure 2.21. The region to the left of $A B$ represents the potential energy well equivalent to the metal surface. The lines represent the continuous energy levels of the metallic electrons and the spacing of the lines is an indication of the density of the quantum levels. The region to the right of $A B$ depicts the potential energy well representing the mercury atom and shows an exclted level and the ground level of the atomic electrons. The symbols used in the diagram are defined as follows:

$$
\begin{aligned}
& M=\text { energy of potential well representing the metal } \\
& \varnothing=\text { work function of the metal }
\end{aligned}
$$




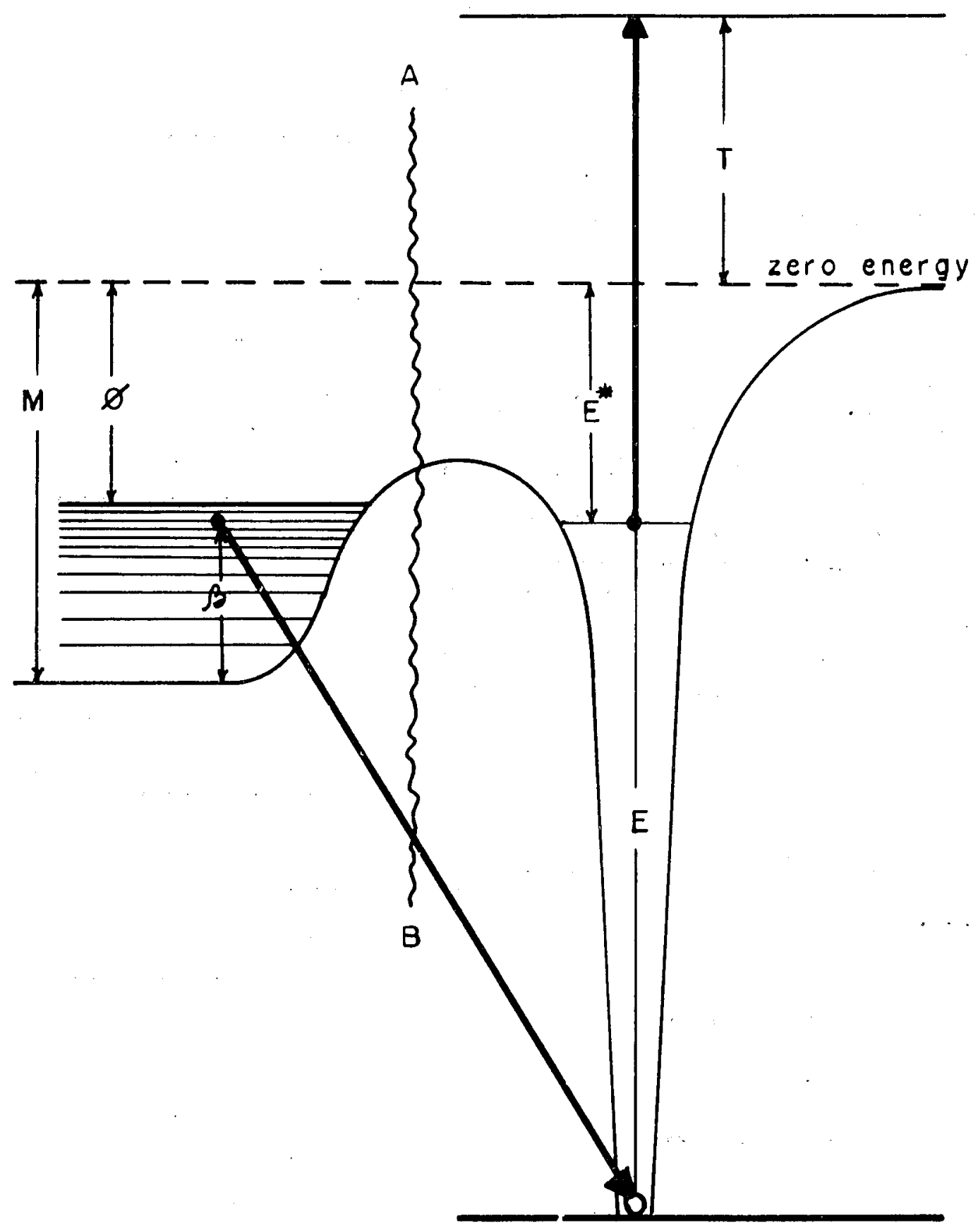

FIG. 2.21 Energy Level Diagram of Excited Atom and Metallic Electrons 


$$
\begin{aligned}
& \boldsymbol{B}=\text { kinetic energy of metallic electron } \\
& E^{*}=\text { energy of excited state } \\
& E=\text { energy difference between excited and ground state } \\
& T=\text { kinetic energy of ejected electron }
\end{aligned}
$$

A common zero of energy for the metal surface and atomic electrons is taken as shown. The heavy dots represent electrons, while the circle represents an electron vacancy. Electron emission occurs when a metallic electron fills the vacancy in the ground state level of the atom and the excited electron leaves the atom. From this analysis it is seen that the excited atomic electron rather than the metallic electron is the one ejected. The two electron transitions are represented by the heavy arrows. This secondary emission process is commonly referred to as Auger de-excitation and can be worked out rigorously if the wave functions of the two electrons are known. A simple energy balance for the process takes the form:

$$
\begin{aligned}
& \left\{\begin{array}{l}
\text { Energy to free atomic } \\
\text { electron and impart to } \\
\text { it a kinetic energy } T
\end{array}\right\}=\left\{\begin{array}{l}
\text { Energy required to trans- } \\
\text { fer a metallic electron } \\
\text { to atomic ground state }
\end{array}\right\} \\
& (E q .2 .2 I) \quad E^{*}+T=\left(E+E^{*}\right)-(M-\beta)
\end{aligned}
$$

and

$$
\text { (Eq. 2.22) } T=E-M+\beta
$$


If the metallic electron is at the surface of the electron sea then $(M-\beta)=\varnothing$ and:

$$
\text { (Eq. 2.23) } \quad T=E-\varnothing
$$

The condition for electron emission is therefore $E \geq \varnothing$. 
CHAPTER 3

\section{Experimental Procedure}

\subsection{Experimental Apparatus}

The experimental tube, represented schematically in figure 3.II, may be broken down into three distinct sections for the purpose of analysis. Section I consists of the arcdischarge tube which provides a source of positive mercury ions. Its construction is of Pyrex glass, blown to the proper dimensions, and fitted with four electrodes. The first electrode, $\mathrm{V}_{\mathrm{g}}$, acts as a ground point for the mercurypool cathode as well as an anchor point for the characteristic cathode spot. A high voltage applied to $V_{S}$, by means of an induction coil, produces a spark between this electrode and the cathode, and in this manner the arc discharge is initiated. Once the arc is started, a discharge of constant intensity is maintained between the primary anode, $V_{a}$, and the cathode. By applying a potential to the fourth electrode, $V_{b}$, a discharge, whose intensity can be controlled, is set up between $V_{b}$ and the cathode. The d.c. potentials are applied to $\mathrm{V}_{\mathrm{a}}$ and $\mathrm{V}_{\mathrm{b}}$ through current limiting resistors by a d.c. generator. The current to the respective 


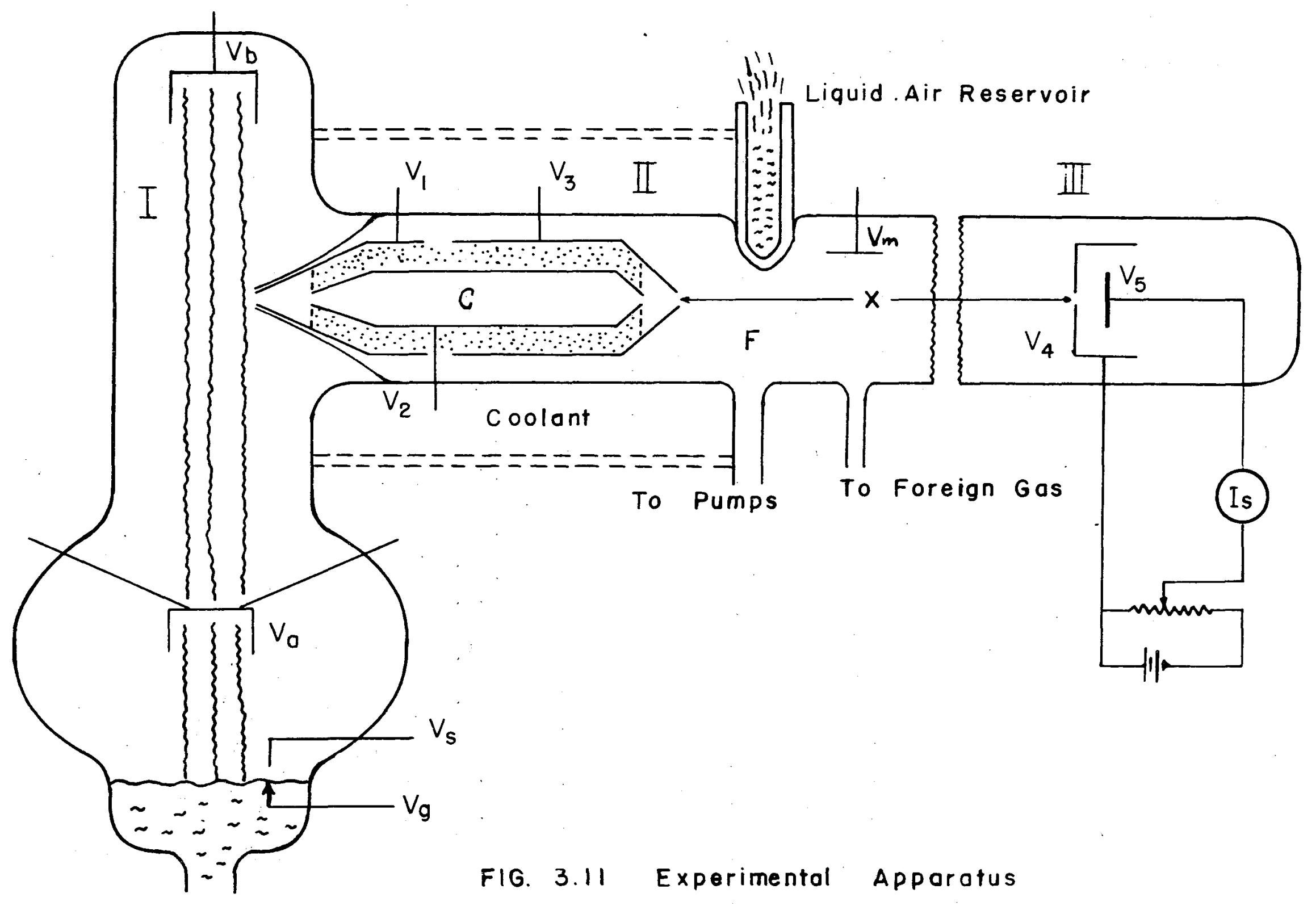


anodes may be varied by means of these resistors. However, since the mercury arc discharge tube is a constant potential device, the voltages measured at $V_{a}$ and $V_{b}$ remain approximately constant (not necessarily equal) irrespective of the applied potential from the generator. $\left(V_{a}\right.$ and $V_{b}$ are of course less than the applied generator voltage in any case.)

The second region of the experimental tube contains the electrodes responsible for extracting and accelerating the ions produced in the discharge. In addition this section is provided with a charge-transfer chamber and an electrode for stopping the positive ions, which escape charge transfer, from reaching the target. The electrode assembly extends partially into the plasma and consists of three tapered and concentric cylinders insulated from one another as shown.- The configuration is supported in a glass cylinder, which is attached to the discharge tube, by means of three leads sealed through the glass as illustrated. The nose of $V_{1}$ extending into the plasma is surrounded by a tapered glass tube which is ring sealed into the containing glass cylinder. The entire glass cylinder surrounding the electrode region is incased in a Styrofoam jacket of insulation which may be filled with a coolant consisting of a dry-1ce and acetone mixture. 
Positive ions are extracted from the plasma by means of a high negative potential applied to $V_{1}$. These ions may then be accelerated by applying a higher negative potential at $\mathrm{V}_{2}$. The fast ions then enter the field-free chargetransfer region $C$ which contains a few drops of mercury liquid to provide the required vapor pressure for charge exchange. The neutral atoms formed in $\mathrm{C}$ continue through electrode $\mathrm{V}_{3}$ toward the target, while the remaining lons are stopped by a positive potential on $V_{3}$. The small perforations in the tapered ends of the cylindrical electrodes provide a well collimated beam of neutral atoms at the target. These small openings also serve to drastically reduce the pumping speed at the charge-transfer chamber and thus maintain the higher vapor pressure required in this region.

Region III is comprised of the target and collector assembly. The collimated beam of neutral atoms. passes through the circular aperture in the cylindrical collector and strikes the enclosed target. The ejected electrons from the target are then accelerated toward the surrounding collector by a suitably applied electric field between $V_{4}$ and $V_{5}$

In order to test the hypothesis that the neutral atoms formed in the charge-transfer process are metastable, It is necessary to vary the distance of the target from the 
charge-transfer chamber. This is accomplished by means of the apparatus 1llustrated in figure 3.12. The collector is mechanically fastened to the target assembly with the aid of an insulating material. The target plate is itself readily removable to facilitate the study of various target materials. Three rods, $r$, are fastened $120^{\circ}$ apart to the target base $B_{I}$ and extend through an anchor-cylinder, $A_{I}$, to a second base, $B_{2}$. The motor rotor, $M$, is attached to $B_{2}$ by means of ball bearings in such a manner that its rotation does not induce $B_{2}$ to rotate. The connection, however, is such to permit the rotor either to pull $B_{2}$ towards $A_{2}$ or push it towards $A_{1}$. A threaded shaft, $R$, extends through the rotor and is fastened at each end to anchor-cylinders $A_{1}$ and $\mathrm{A}_{2}$ which are snugly fitted to the glass walls. The inside of $M$ is also threaded to mate with $R$. Now, when the motor stator is passed around the glass tube and moved to the vicinity of $M$, the rotor turns on the threaded shaft moving either towards $\mathrm{A}_{1}$ or $\mathrm{A}_{2}$ depending. upon the polarity of the stator voltages. As it does so, the target is pulled toward $A_{2}$ or pushed toward $A_{1}$ as a result of the ball bearing coupling. The electrical connection to the target is made to $\mathrm{A}_{2}$ by a wire sealed through the glass. The connection to the collector is accomplished by means of a spring clip attached to the collector which rides on a rall mounted along the bottom of the glass tube. A lead to this 


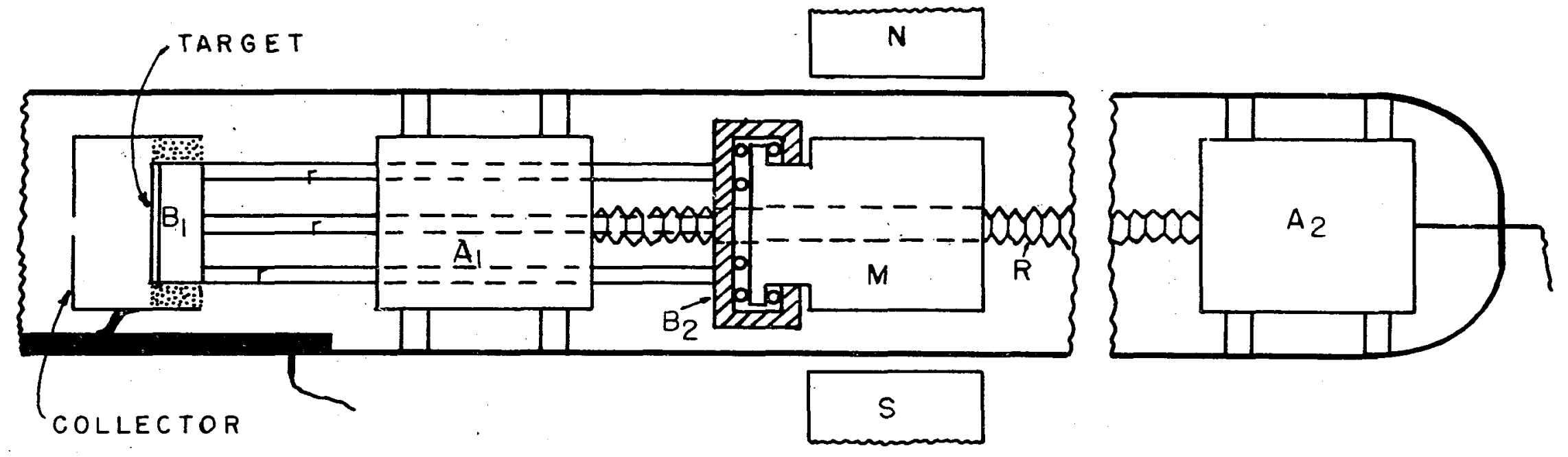

FIG.3.12 Target Receding Apporotus 
rail is also sealed through the glass. In this manner the distance between the target and the charge-transfer chamber may be varied over a range of about $40 \mathrm{~cm}$.

The pressure between the charge-exchange region and the target, as monitored by an ion gauge, is maintained at about $10^{-6} \mathrm{~mm}$ by means of a mechanical fore pump in series with two mercury diffusion pumps in sertes with each other. The diffusion pumps, which are connected to this region, F, through a liquid-air trap, are further aided by a double walled glass tube, filled with liquid nitrogen, which protrudes into region $F$. Such a vacuum is extremely good for a system exposed to an open source of mercury. The pressure In the charge-transfer chamber is not known accurately but presumed to be of the order of $10^{-3} \mathrm{~mm}$ as the presence of mercury droplets should assure nearly saturation vapor pressure in this region.

An opening into region $F$ is provided through which foreign gases may be admitted to quench the anticipated metastable atoms. Minute quantities of these gases are admitted by means of a variable leak valve which is in series with the external gas source and region $F$.

The material used in the discharge tube electrodes, $V_{a}$ and $V_{b}$, as well as that of the target electrodes, $V_{4}$ and $\mathrm{V}_{5}$, is nickel which has been machined to the proper 


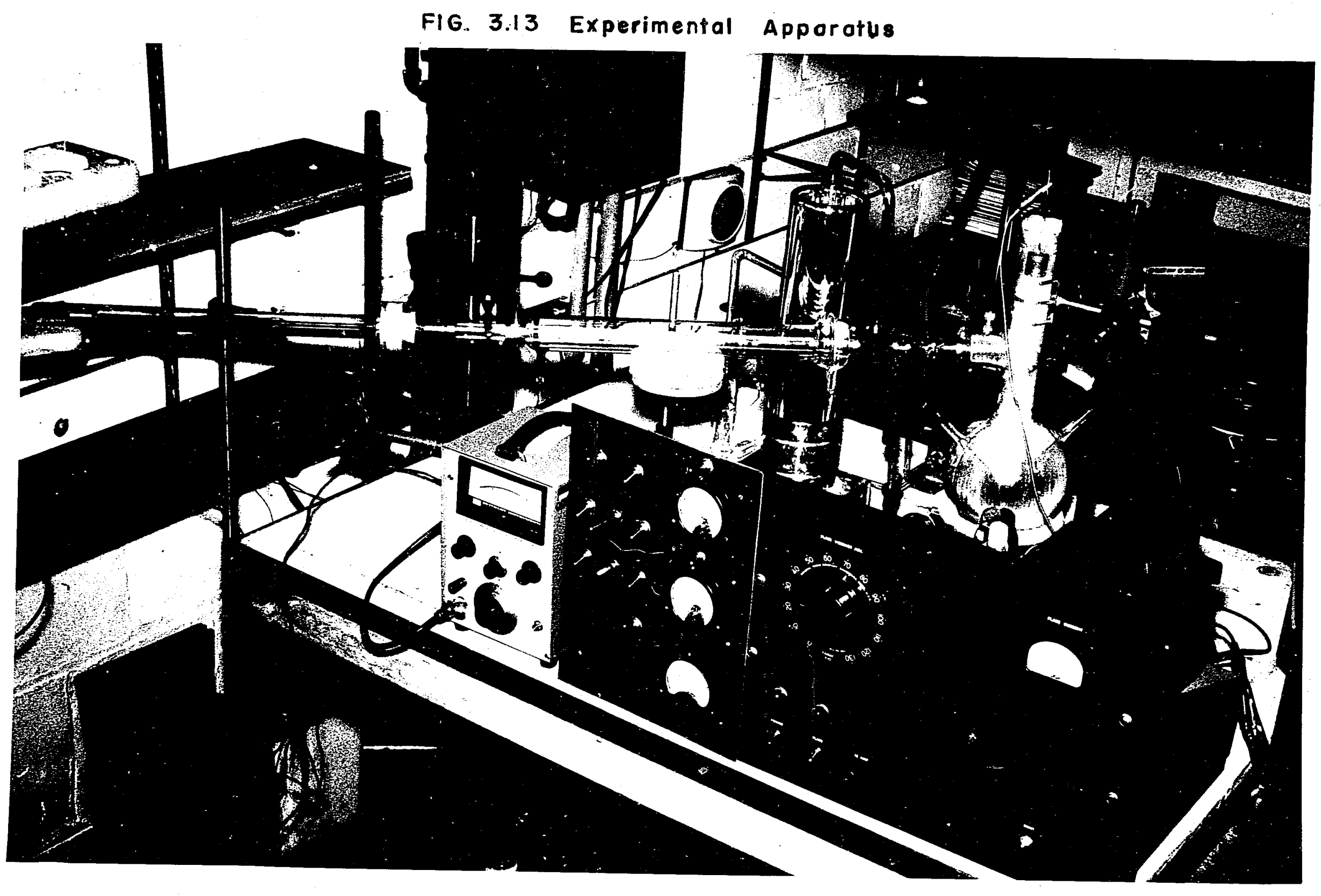


dimensions. The electrodes $V_{1}, V_{2}$, and $V_{3}$ are all stainless steel. Nelther of these metals amalgamates with mercury. The purpose of electrode $V_{m}$ will be explained at a later time.

The overall apparatus setup, including the discharge tube, the time-of-flight tube, and various meters and power supplies is shown in figure 3.13 .

3.2 Experimental Data

Preliminary tests with the apparatus showed that the secondary emission from the target increased rapidly as the current to anode $V_{b}$ was increased from zero to about 1.5 amps. Any further increase in the current did not appreciably influence the secondary emission. The secondary electron current was also found to vary directly with the intake potential; $V_{1}$, over the range of 0-5000 v. The accelerating electrode, $V_{2}$, was not as effective as desired, due to the tendency of the dielectric between $v_{1}$ and $v_{2}$ to break down when the potentials between these electrodes differed greatly. This effect caused the potential on $V_{I}$ to increase as that on $\mathrm{V}_{2}$ increased. 
It was also discovered early in the experiment that if the potential on the collector, $V_{4}$, was more negative than that on the target, $V_{5}$, the meter in the target circuit read a negative current. (figure 3.21 ) with this polarity between target and collector, secondary electrons could not leave the target. The inference, then, was that negatively charged particles were reaching the target along with the neutral atoms. This negative current was very large and actually approached the magnitude of the secondary emission current. A logical explanation, and one used by earlier researchers, would be that the neutrals were reflected from the target and struck the collector. Secondary electrons ejected in this manner from the collector would then be free to reach the target. However, it seemed unlikely that the reflection would be so intensive. A second explanation seemed to be that a high energy, well-collimated beam of negative ions or electrons was reaching the target. To test this theory, a magnetic field of about 200 gauss was provided, perpendicular to the beam of neutrals at a point just beyond the exit of the charge-transfer chamber. This field greatly reduced the negative current at the target but did not eliminate it altogether. An electrode, $V_{m^{y}}$ (figure 3.11) held at a high positive potential (1000 v) and placed parallel to the beam between the magnetic field and the target, served to eliminate the unwanted negative current completely. 

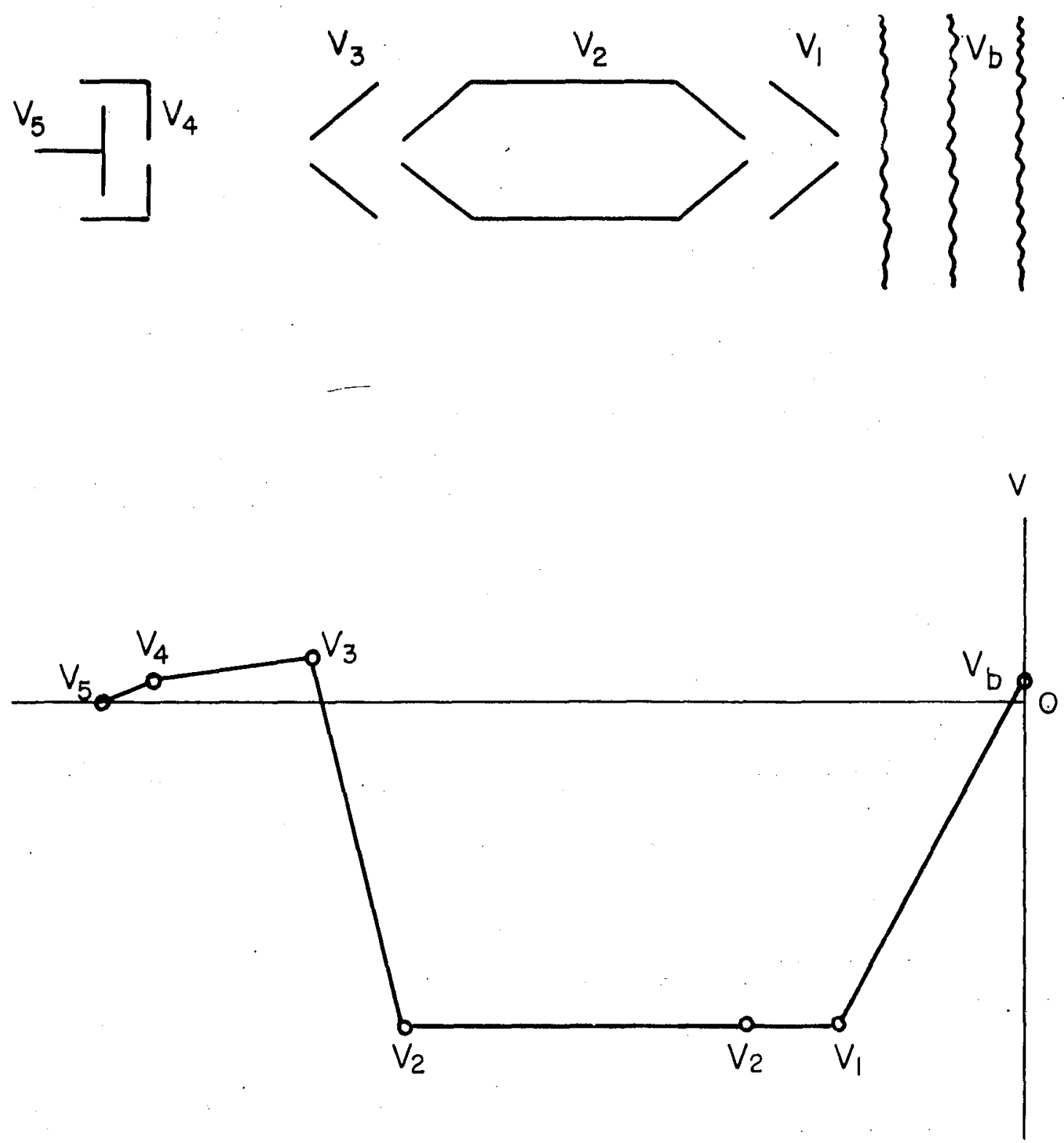

FIG. 3.21 Potential Distribution 
It was thereby assured that only the secondary electrons, ejected from the target by neutral atoms, were responsible for the target current. That is, with the collector more negative than the target, the target current was zero.

Since the magnetic field alone would have been more than ample to deter a beam of electrons from the target, and since there was no logical means for a beam of fast electrons to form, the assumption was made that the negative particles were atomic mercury ions. The energy of these Ions was measured by varying the potential on the target from zero to some negative value which just stopped the ions from reaching the target. During this process, the collector was always held more negative than the target so that no secondary electrons could leave the target. The very fact that the negative ions could be stopped was a good indication of their existence. A plot of this stopping potential, $V_{s}$, as a function of the intake potential, $V_{I}$, is shown in figure 3.22. Unless otherwise specified it may be assumed that $V_{2}$ was held equal to $V_{1}$ throughout the experiment.

The efficiency of the neutral atoms to Ilberate secondary electrons is measured by the ratio, $\boldsymbol{\gamma}$, of the secondary electron current to the incident neutral atom current. The secondary electron current could be read directly from a meter in the target circult but determining the incident 


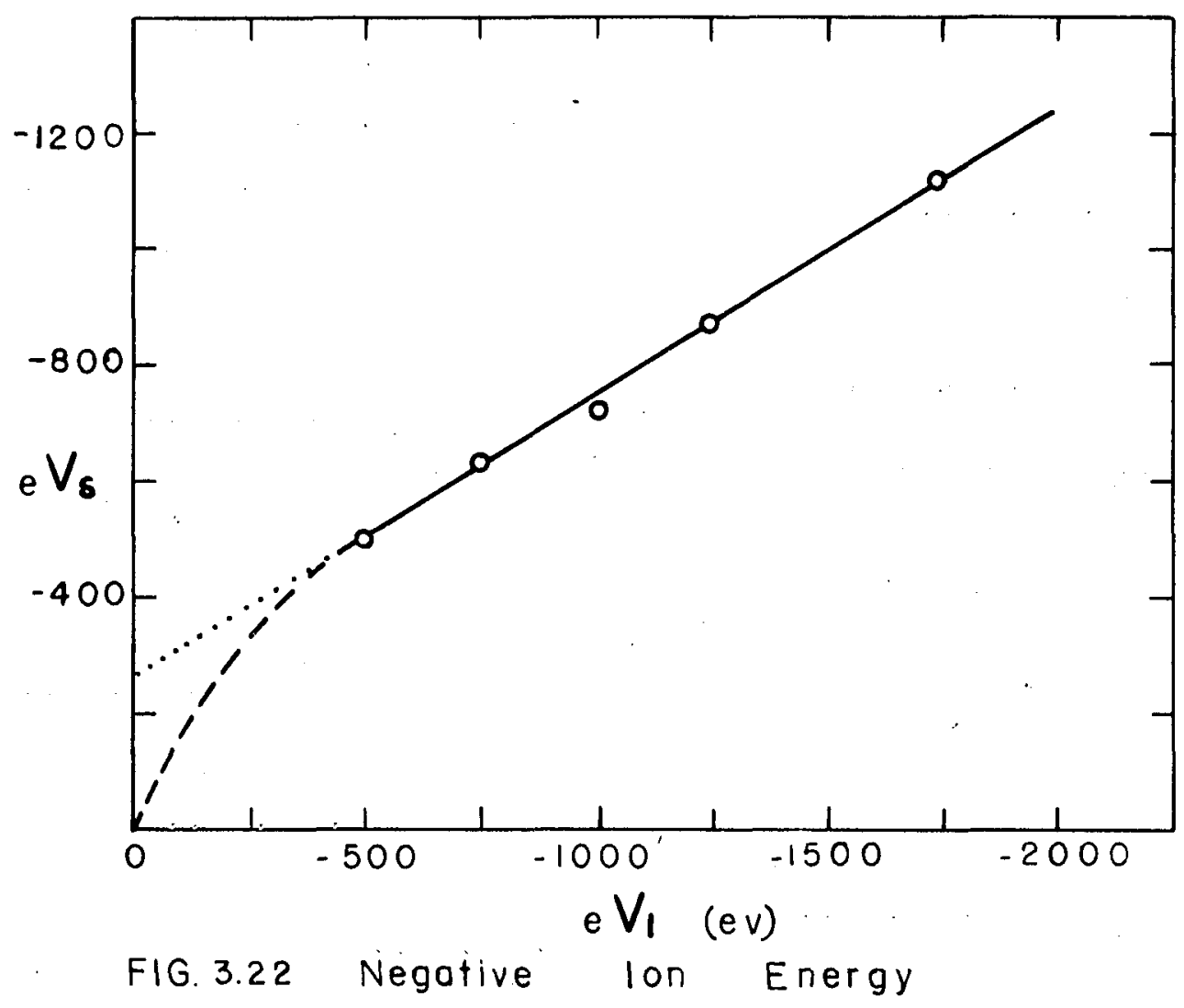


neutral current was not so readily achieved. The following method to this end was adopted. With the positive ion retarding electrode, $V_{3}$ and the target, $V_{5}$, at the same potential as $V_{1}$ and $V_{2}$, positive ions as well as neutral atoms were allowed to strike the target. A collector $\left(v_{4}\right)$ slightly more negative than the target prevented secondary electrons ejected by the neutrals (or by the ions) from leaving the target. Negative ions were prevented from reaching the target in this instance, as well as in all further work, by means of the magnetic and electric field described in the preceding paragraphs. Thus, under this potential distribution, the meter in the target circuit read a positive current $I_{W}^{+}$due to the impinging positive ions. By cooling the charge-exchange region with a dry-ice-acetone mixture in the styrofoam jacket, the pressure in the chargeexchange region was reduced to a value below the chargetransfer threshold. The positive ion current read at the target under these conditions was increased to a new value $I_{c}^{+}$. This increase could be attributed to the positive ions which had previously undergone charge transfer but which now were striking the target. The difference between these two currents $\left(I_{c}^{+}-I_{w}^{+}\right)$would then represent the neutral atom current responsible for the secondary emission at the target with the charge-exchange chamber at ambient temperature. If $I_{s}$ represents the secondary electron current and $I_{n}=I_{c}^{+}-I_{w}^{+}$, 


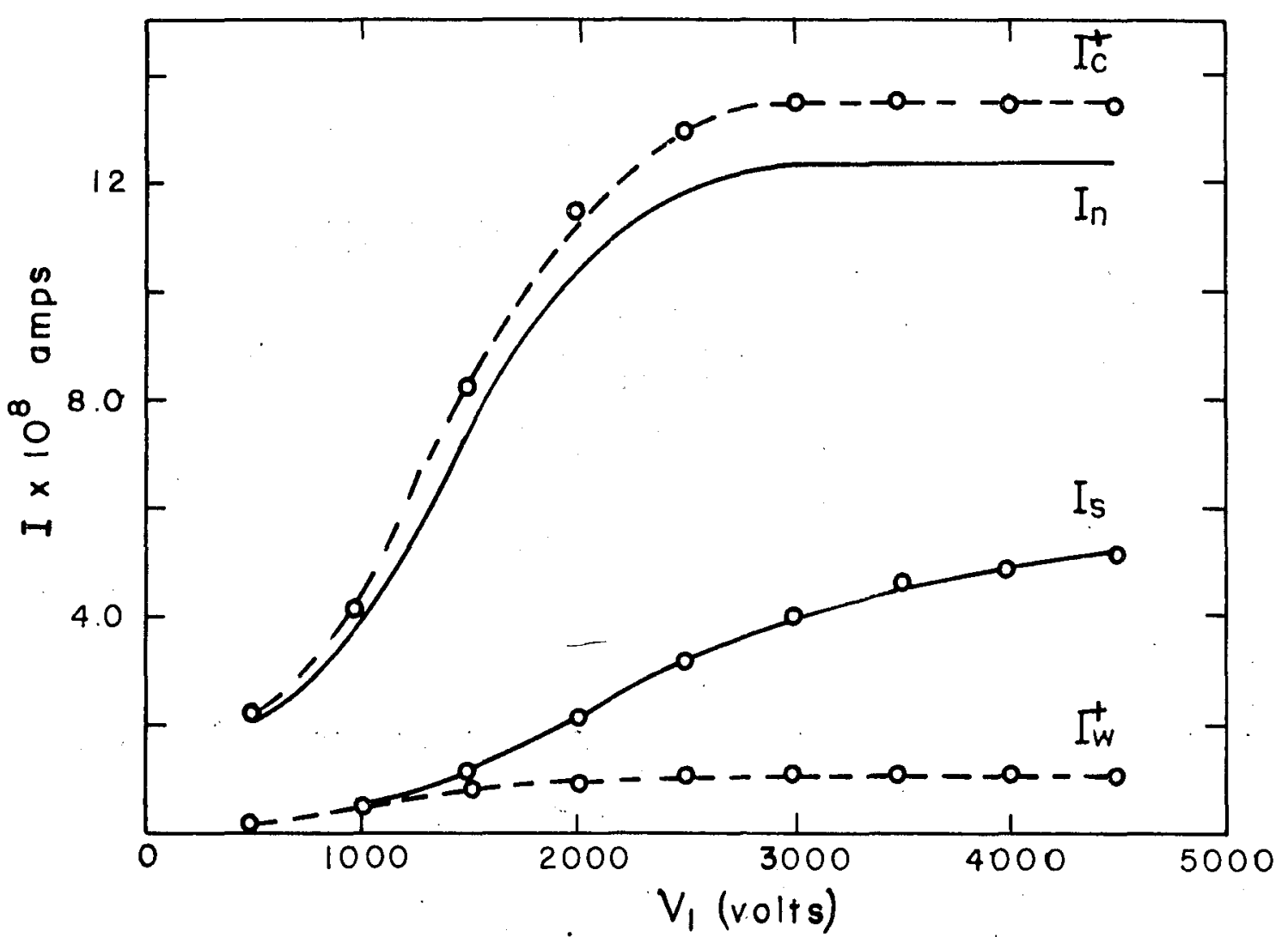

FlG. 3.23 Gurrents Used to Find $\gamma$

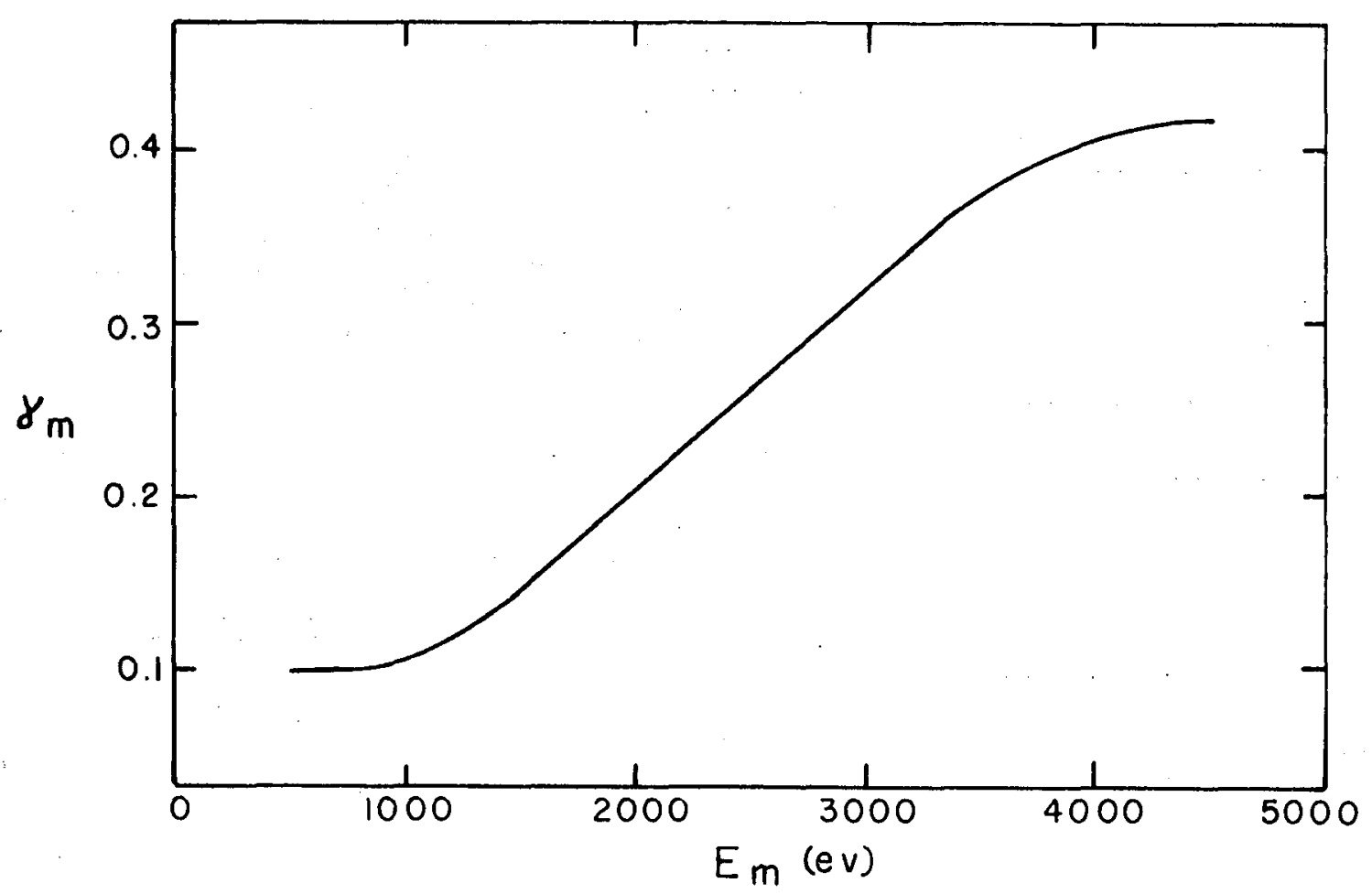

FIG. $3.24 \gamma_{m}$ vs Metastable Kinetic Energy 
then the ratio of the secondary electron current to the primary neutral atom current is given by:

$$
\gamma=\frac{I_{s}}{I_{n}}=\frac{I_{s}}{I_{c}^{+}-I_{W}^{+}}
$$

The values of $I_{n}, I_{s}, I_{c}$, and $I_{w}$ as a function of intake potential $\left(v_{1}\right)$ are plotted in figure 3.23. The value of $\gamma$ as a function of intake potential is plotted in figure 3.24 .

In order to test the hypothesis that the neutral atoms formed in the charge-transfer process were metastable, the distance between the target and charge-exchange region was varied in the manner previously described. As this distance increased, the secondary emission from the target decreased in a manner which indicated that the neutrals were excited and were decaying in flight. The secondary electron current as a function of target distance, $x$, is plotted in figures 3.25 and 3.27 for various values of $V_{1}$. The distance $x$ is taken as that from the exit of electrode $v_{3}$ to the surface of the collector electrode, $V_{4}$. Since it is only the ratio of secondary electron currents at various values of $x$ which are important, the $x=0$ point is not critical nor is the fact that the collector surface is slightiy forward of the target. The flight time may be computed from the flight distance $(x)$ if the speed of the neutral atoms is known. 


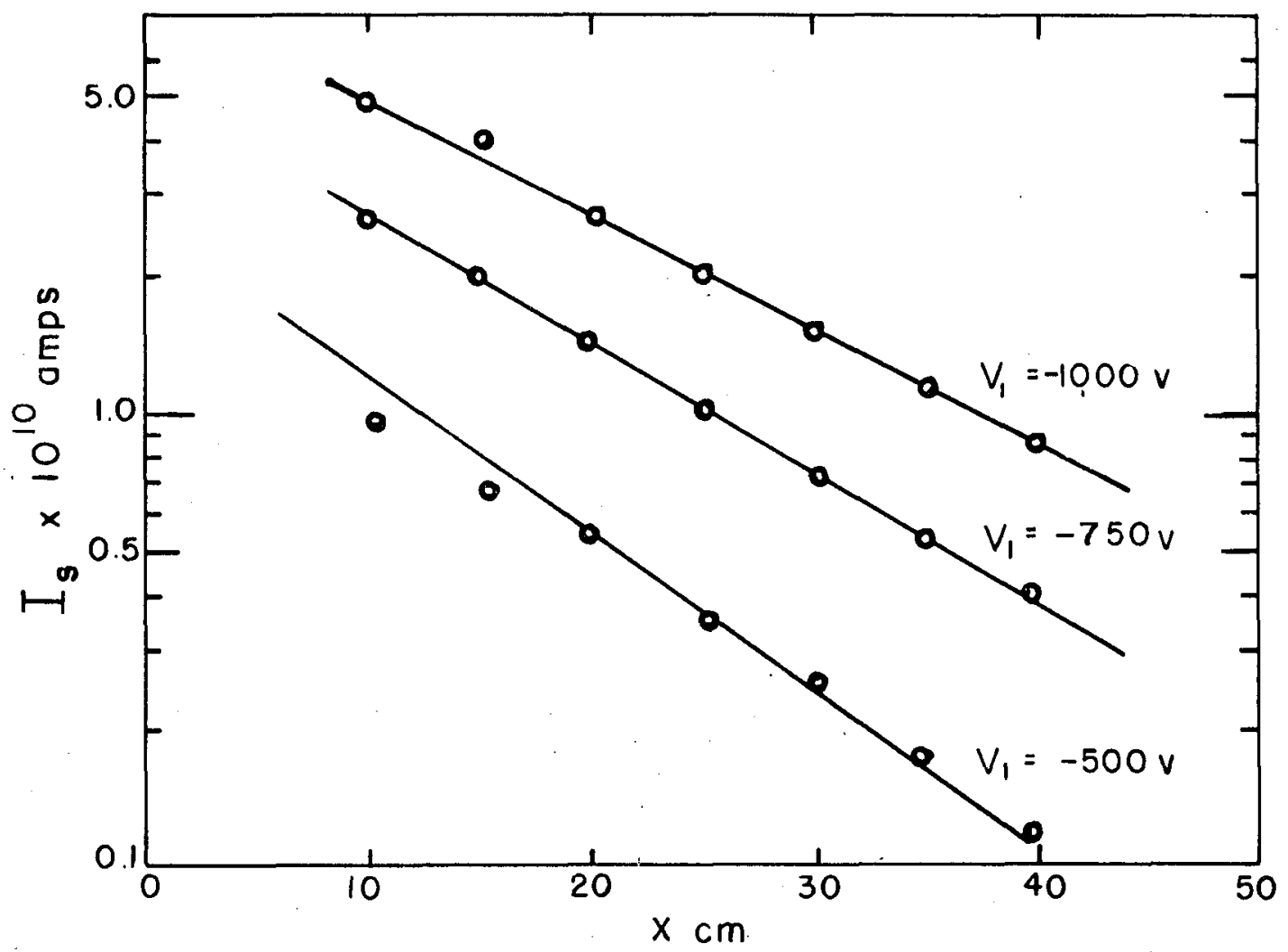

FIG. 3.25 Secondary Current vs Flight Distance

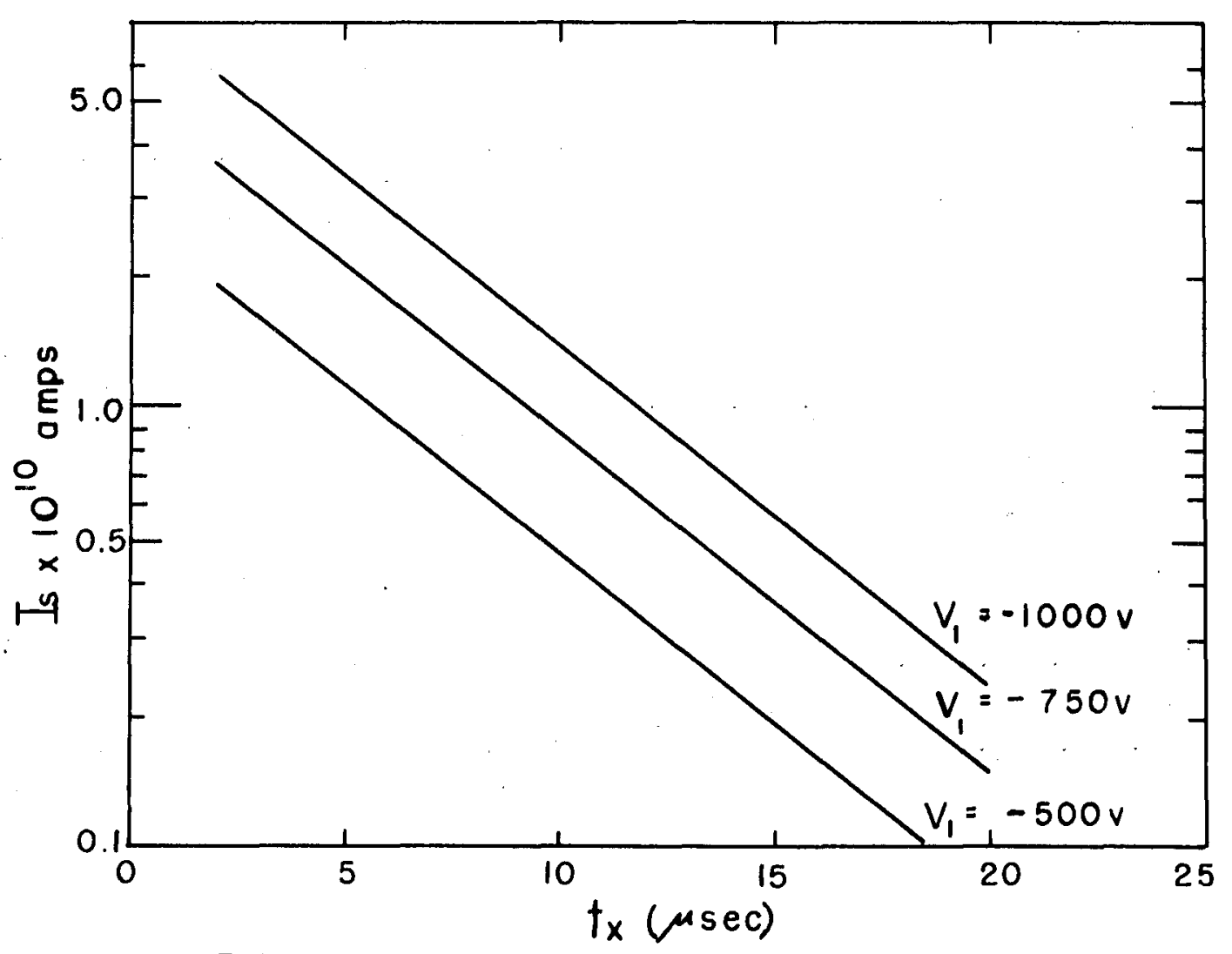

FIG. 3.26 Secondary Current vs Flight Time 


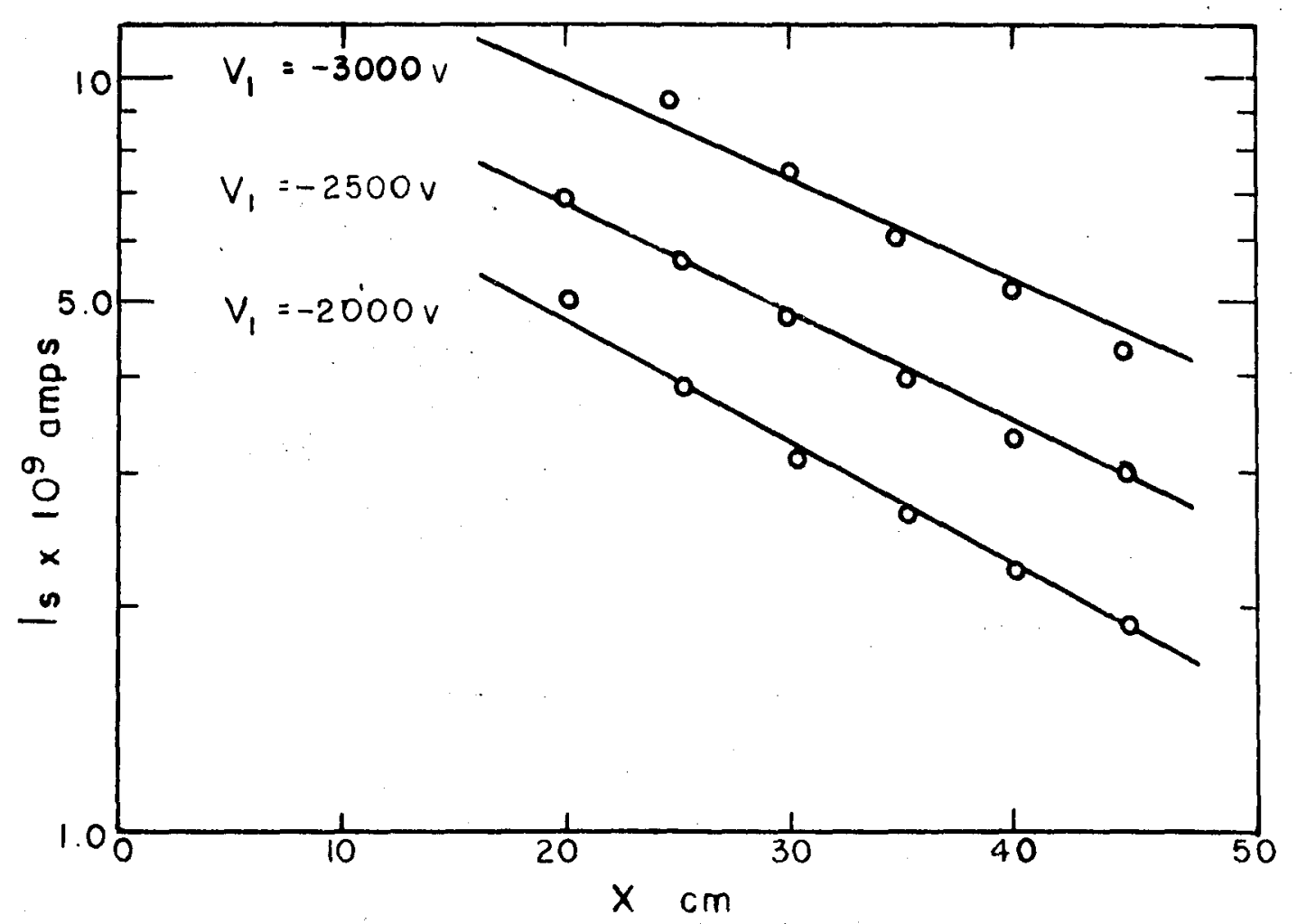

FIG. 3.27 Secondary Current vs Flight Distance

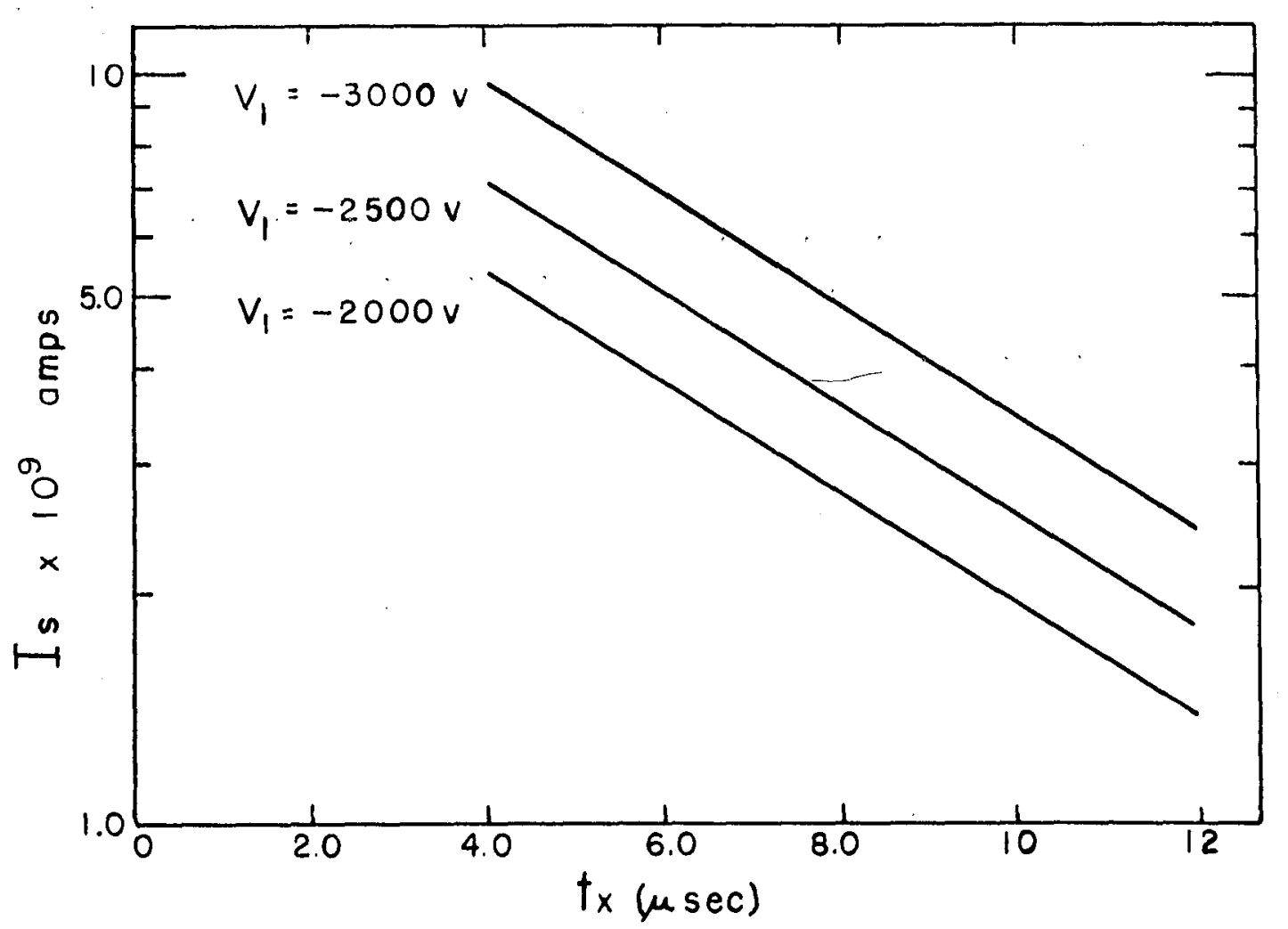

FIG. 3.28 Secondary Current vs Flight Time 
This speed is found from the expression:

$$
v=\sqrt{\frac{2 \cdot Q \cdot E}{M}}
$$

where: $Q=$ charge on mercury ion

$$
\begin{aligned}
& E=\text { Ion accelerating potential } \\
& M=\text { mass of mercury ion }
\end{aligned}
$$

The flight time is then computed from the relation $t=x / v$. Figures 3.26 and 3.28 show the secondary emission as a function of flight time.

In order to ascertain that the secondary emission was not a photo-electric effect due to the radiation from the mercury arc, the secondary electron current as a function of distance was examined with the intake potential equal to zero. The secondary emission under these conditions vanished at all distances.

The second method of quenching the presumed metastables, consisted of admitting, in turn, minute quantities of hydrogen and argon gas into the flight path of the neutrals. Hydrogen was selected for Its low mass and number of degrees of rotational freedom, while argon was chosen for its inert character. A collision of a mercury atom with hydrogen would produce little deflection of the fast, heavy, mercury atom. The rotational energy, however, could easily be 


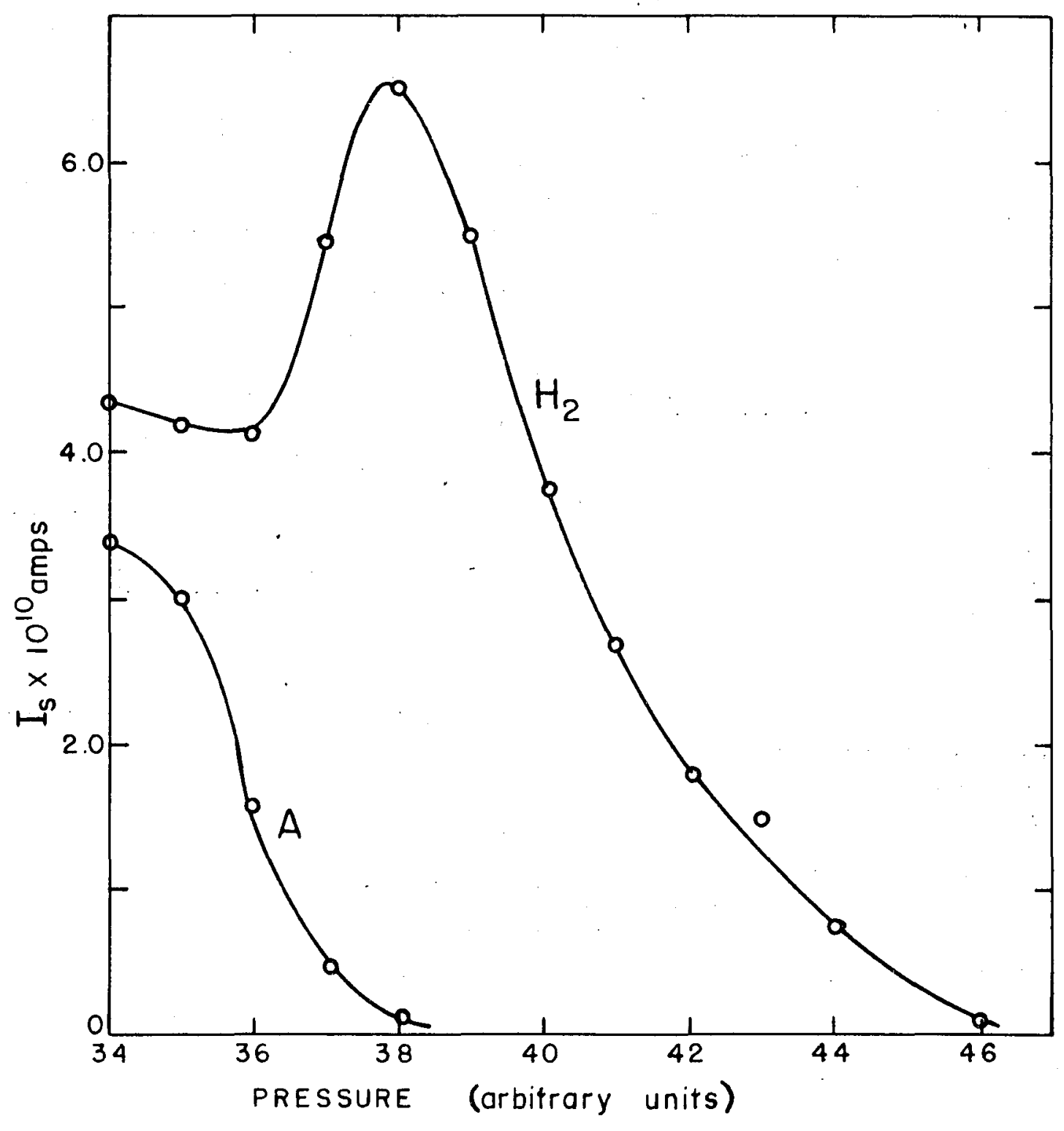

FIG. 3.29 Secondary Current vis Pressure 
transferred to the metastable atom, resulting in its de-excitation. There also exists the possibility of the formation of positive hydrogen or mercury ions, in the collision process, which would reach the target. The secondary emission, contrasting the effects of hydrogen and argon, is plotted as a function of pressure in figure 3.29. The pressure referred to is that between the target and charge-exchange region.

In view of the overwhelming evidence that the neutral atoms were metastable, an attempt was made to determine their energy by plotting the secondary electron energy spectrum. This was accomplished by varying the collector potential from $-40 \mathrm{v}$ to $+40 \mathrm{v}$ and monitoring the secondary electron current. The value of secondary electron current saturated well before these limiting values were reached. The negative value of the potential on the collector at which the secondary emission just became zero represented the maximum kinetic energy of the electrons leaving the target. Typical secondary electron energy spectra are illustrated in figures 3.210 and 3.211. An energy spectrum along with the corresponding energy distribution for the secondary electrons is shown in figures 3.212 and 3.213 . 


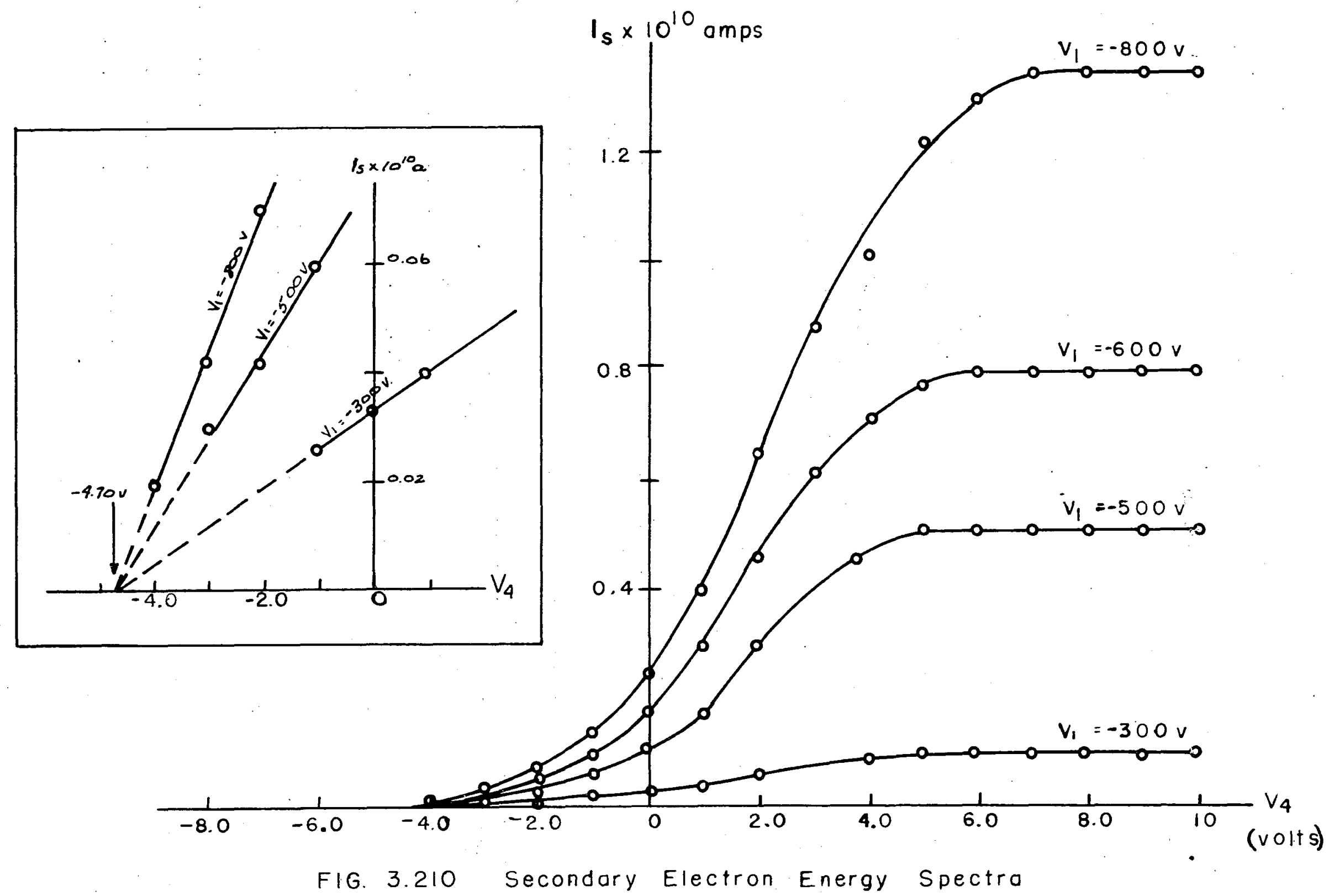




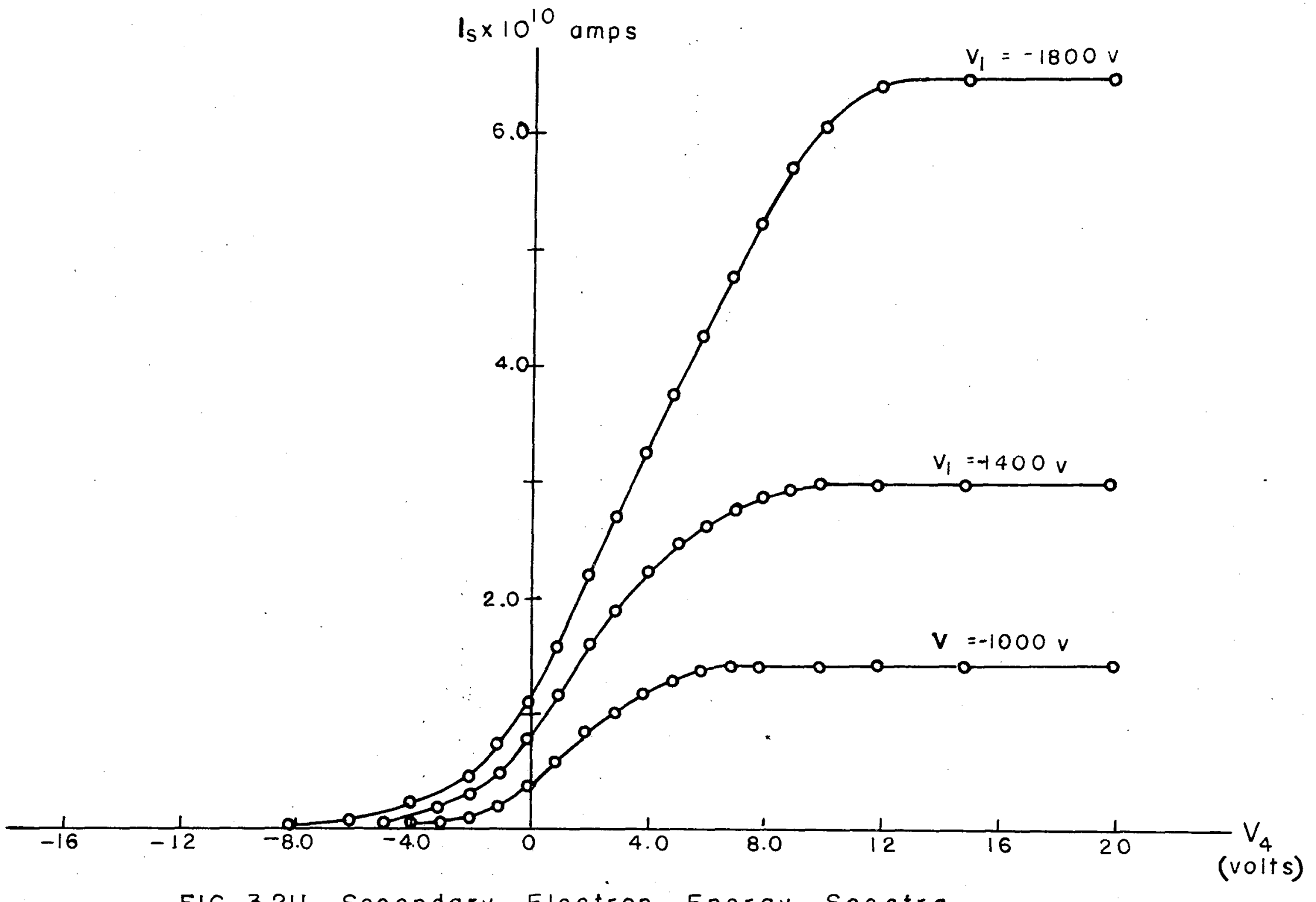

FIG. 3.2II Secondary Electron Energy Spectra 
Typical values of potentials and currents at various electrodes, recorded in the course of the experiment, are tabulated below:

$$
\begin{aligned}
& \mathrm{V}_{\mathrm{g}}=\operatorname{arc} \text { cathode potential............. } 0.0 \mathrm{v} \\
& \mathrm{V}_{\mathrm{a}}=\text { Ist arc anode potential............. } 11.0 \mathrm{v} \\
& \mathrm{V}_{\mathrm{b}}=\text { 2nd arc anode potential............ } 21.0 \mathrm{v} \\
& I_{a}=I \text { st arc anode current.............. } 2.0 \mathrm{a} \\
& I_{b}=\text { 2nd arc anode current............ } 0.5 \mathrm{a} \\
& \mathrm{V}_{1}=\text { intake potential................. } 0-3000 \mathrm{v} \\
& \mathrm{V}_{2}=\text { accelerating potential............ } 0-3000 \mathrm{v} \\
& \mathrm{v}_{3}=\text { positive ion retarding potential...... } 300 \mathrm{v} \\
& \mathrm{V}_{\mathrm{m}}=\text { negative ion collecting potential...... } 1000 \mathrm{v} \\
& B=\text { negative ion deflecting field........200 gauss }
\end{aligned}
$$

$$
\begin{aligned}
& \mathrm{V}_{4}=\text { collector potential............... } 40-+40 \mathrm{v} \\
& \mathrm{v}_{5}=\text { target potential................ } 0.0 \mathrm{v} \\
& I_{S}=\text { secondary electron current......... } 10^{-9} \text { a }
\end{aligned}
$$

All potentials except $V_{1}, V_{2}$, and $V_{4}$ were kept at the values indicated during normal operations. $v_{1}$ and $v_{2}$ were the same at all times unless specified otherwise. $V_{4}$, except during the taking of the secondary electron energy data, was kept at a value to insure operation in the saturated regions of the energy spectrum curves. In recording values of secondary 


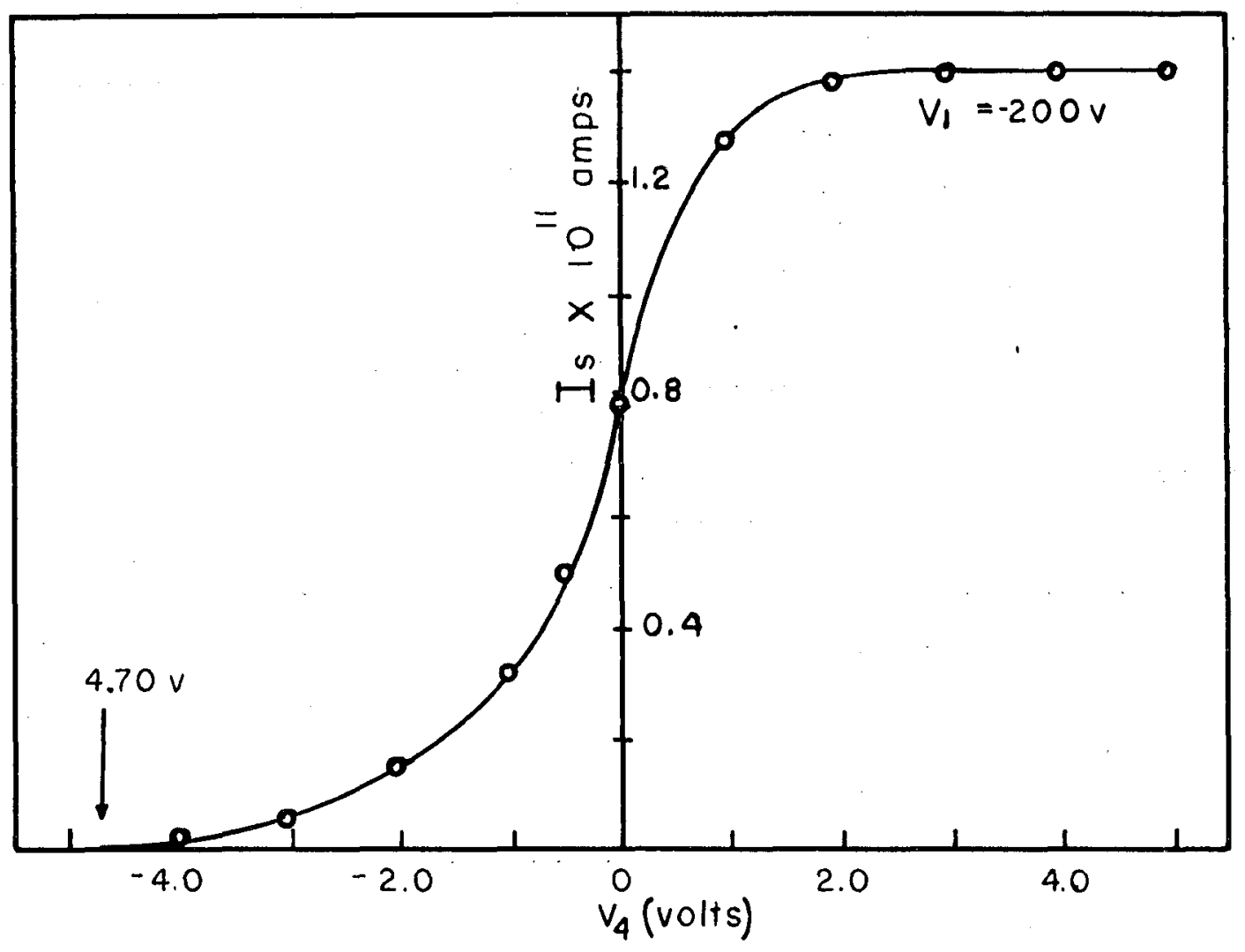

FIG. 3.212 Secondary Electron Energy Spectrum

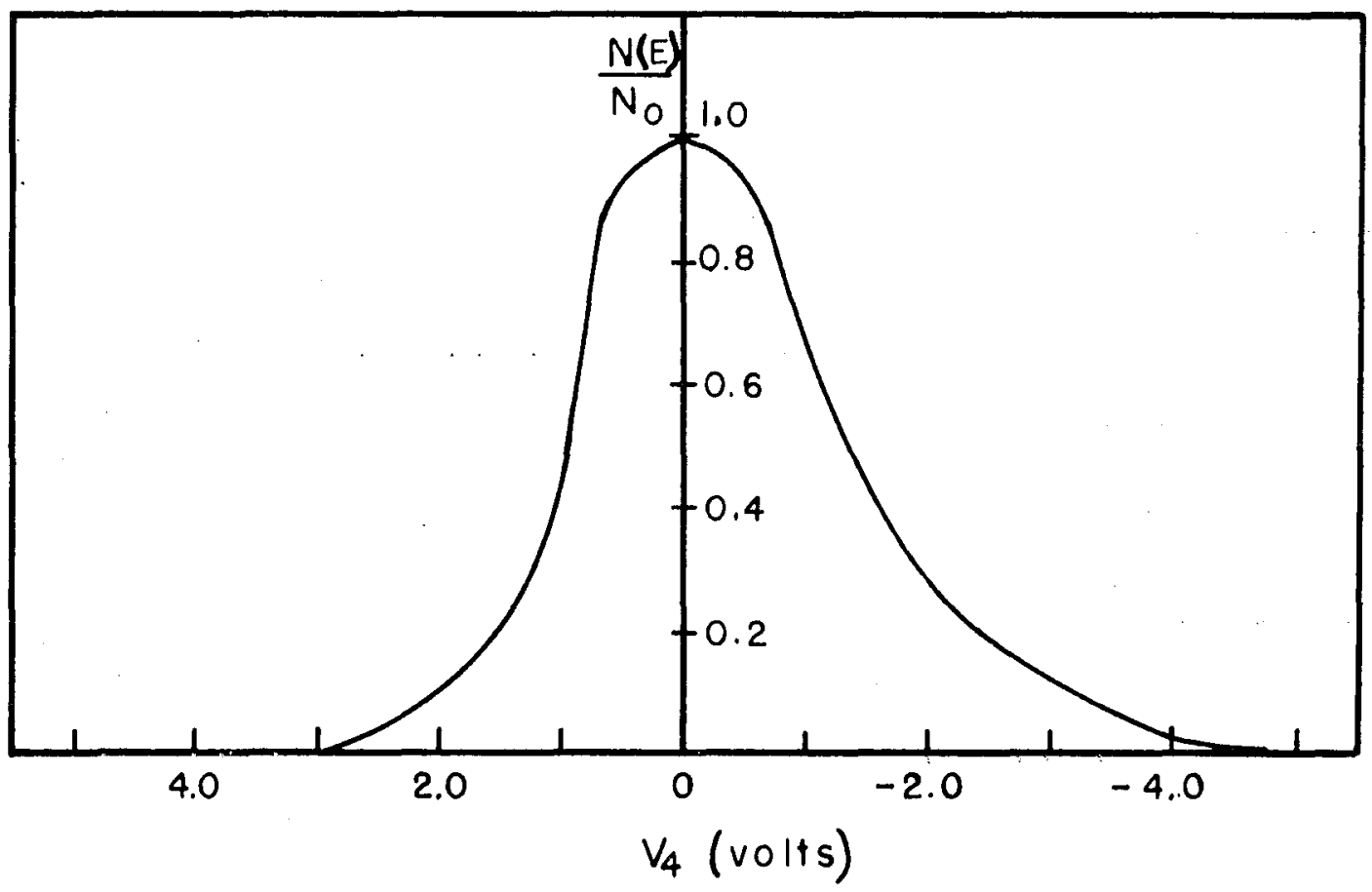

FIG. 3.213 Secondary Electron Energy Distribution 
electron current, the magnetic field, $V_{3}$, and $V_{m}$ were always used to prevent unwanted ions from reaching the target. AII switches, wires, batteries and meters used were well shielded and insulated where necessary.

A thorough discussion of the data given here will be presented in the following chapter. 
CHAPTER 4

Analysis of Results

The foregoing data of chapter 3 will now be subjected to a detailed analysis and discussion. Much of the work of earlier researchers in the field is substantiated by these data; some work is contradicted. Of primary importance is the fact that the metastable hypothesis is overwhelmingly verified.

\subsection{Negative Ion Formation}

Although the investigation of electron attachment by mercury atoms was not the intended purpose of the present work, this effect presented itself as one to be eliminated in order to enhance the success of the experiment: The question of whether or not an atomic mercury atom will attach an electron, thus forming a negative ion, has long been the subject of controversy. The literature contains the reports of investigations both supporting and refuting the occurrence of this phenomenon. Experiments of Arnot and 
Milligan ${ }^{l}$ led them to believe that they had succeeded in forming $\mathrm{Hg}^{-}$Ions. The theoretical work of Massey and $\mathrm{Smith}^{2}$ also predicted the formation of negative ions. Sloane and Press ${ }^{3}$, using mass spectographs, were unable to detect this ion.

In the present work, the question of $\mathrm{Hg}^{-}$ion formation presented itself during the course of eliminating unwanted negative particles from the beam of neutral atoms. The argument in favor of these negative particles being negative mercury ions stems from the following data. The intensity of the negative particle current at the target was relatively large, being of the same order as the secondary electron current. This indicated that the particles were large in number and arrived in a directed beam. Any negative ions formed by atoms of impurities in the system would hardly exist in such large quantities or be collimated. The possibility that the negative particles might be electrons was thought to be equally unlikely since none of the processes by which electrons could be formed would produce a

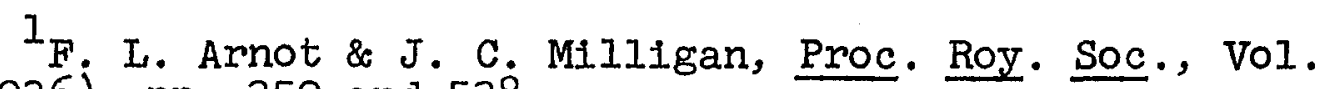
A156 (1936), pp. 359 and 538 .

${ }^{2}$ H. S. W. Massey \& R. A. Smith, Proc. Roy. Soc., Vol. Al55 (1938), p. 472 .

3R. H. Sloane \& R. Press, Proc. Roy. Soc., Vol. Al68 (1938), p. 284 . 
beam of fast collimated electrons. Further, the 200 gauss magnetic field, applied perpendicularly to the beam, would have easily deflected all of the electrons out of the solid angle subtended by the aperture in the collector electrode. This, however, was not the case, as an electric field in addition to the magnetic field was necessary to completely eliminate the negative particles at the target.

For the reasons cited the negative particles are assumed to be $\mathrm{Hg}^{-}$ions. The stopping potential for these lons is shown as a function of the positive ion intake potential $\left(\mathrm{V}_{1}\right)$ in figure 3.22. This stopping potential is actually the potential difference between the positive potential on $V_{3}$ and the negative potential on the target and collector $\left(v_{4}=v_{5}\right)$. Thus an ion must possess a minimum energy of $\mathrm{ev}_{3}$ if it is to reach a target at ground potential. This is Indicated by the intersection of the linear extrapolation of the stopping potential curve with the ordinate at a value equal to $V_{3}$. The ion energy is then the value of $e\left(V_{3}-V_{4}\right)$ at the instant the negative ion current at the target just reaches zero. As previously stated, the potentials $V_{1}$ and $V_{2}$ were set equal, and thus any negative ion formed in the charge-exchange region (or between $v_{1}$ and $v_{2}$ ) would reach the target with an energy $e\left(2 v_{1}+v_{3}\right)$ due to the potential distributions shown in figure 3.21. A negative 1on formed between $V_{2}$ and $V_{3}$ would have an energy between $e V_{1}$ and 
$e\left(V_{1}+V_{3}\right)$. Finally, if the ions were formed between $V_{3}$ and the target their energy would be less than $\mathrm{eV}_{I}$. The final condition is seen to exist in all cases, namely that the ion energy is always less than the initial energy of the primary positive ion. The decrease in the ratio of ion energy to intake potential, with increasing values of intake potential, indicates the ions are being formed nearer the target as $V_{1}$ increases. This decrease is evident in figure 3.22 which shows the plot of stopping potential vs intake potential to be approximately linear over the range of measured values, the slope of this line being one-half.

The collision process which perhaps best explains this negative ion formation is capture by a neutral atom:

$$
\text { (Eq. 4.11) } \quad A+B \rightarrow A^{-}+B^{+}
$$

which in the case of excited mercury neutrals in mercury vapor becomes:

$$
\text { (Eq. 4.12) } \mathrm{Hg}^{*}+\mathrm{Hg} \rightarrow \mathrm{Hg}^{-}+\mathrm{Hg}^{+*}
$$

It is possible for either product in the reaction to be excited. However, it is believed that the secondary emission produced by excited negative ions would nearly equal the ion current. Thus the current at the target would be very nearly zero for excited negative ions. Since the existence of excited positive mercury ions has been established by other 
investigators, the slow positive ion is assumed to carry off the excitation energy.

The preceding observations, of course, do not conclusively establish the existence of $\mathrm{Hg}^{-}$ions but rather indicate a need for further more intensive studies of the subject.

\subsection{Metastable Atom Decay}

The primary means utilized to establish the excitation state of the neutral atoms, formed in the chargeexchange chamber, was to provide an opportunity for them to decay in their flight to the target. As recorded in the data of chapter 3, the intensity of secondary emission decreased markedly as the target receded from the chargeexchange region. This decrease in secondary emission could have been caused in several different ways. If the secondary emission was being produced by radiation from the mercury arc discharge, moving the target might shield it from this radiation. This theory was disproved by setting the intake potential $\left(v_{l}\right)$ equal to zero and observing that the secondary electron current dropped to zero for any target position. 
A second effect which would cause the secondary emission to diminish is geometric dispersion of the beam of neutral atoms leaving the charge-exchange chamber. Due to the dependence of this type of dispersion on the solid angle subtended at the target by the beam, the secondary emission decrease would be expected to vary inversely as the square of the distance from the charge-transfer region to the target; i.e., to obey a $1 / R^{2}$ law. Examination of figures 3.25 and 3.27 reveals that this is not the case since a $1 / R^{2}$ plot on a semi-log scale would not yield a straight line. Furthermore, a well collimated beam of neutral atoms entering a near vacuum would be unlikely to exhibit any appreciable dispersion over the relatively short range encountered here.

Simple elastic scattering collisions between the neutral atoms and the mercury vapor atoms would produce an exponential decrease in the number of neutrals reaching the target, and thus in the secondary emission. This phenomenon is indicated by the curves in figures 3.25 and 3.27 . However, the vapor pressure in the flight-path region has been measured to be of the order of $10^{-6} \mathrm{~mm}$ of mercury. The scattering mean-free path for mercury atoms in mercury at this pressure and room temperature is of the order of 200 meters! It is therefore indeed unlikely that the beam intensity is decreased discernibly by scattering processes. 
Finally, the decrease in secondary emission, with increasing target distance, may be explained by the hypothesis that the neutral atoms were in a long-Iived excited state and a portion of them decayed in flight. The exponential decay curves shown in the semi-log plots of figure 3.25 through figure 3.28 verify this hypothesis. The curves of secondary emission as a function of flight distance were found to be straight lines on the semi-log plots for all values of $v_{1}$. This pattern was unaltered by variations in the arc currents, intake potential, or type of target surface. The corresponding flight time curves are also exponential as evidenced by their straight line semi-log plots. The slopes of these lines represent the average decay distances and decay times respectively. The slopes of the decay time curves are found to be approximately equal for all values of neutral atom kinetic energy shown. The decay distance curves then necessarily have different slopes.

The mean Iifetime of the excited neutral atoms is defined as the time for the beam intensity (and thus also the secondary emission intensity) to decrease by a factor of 1/e. It is computed from the decay curves as follows:

$$
\begin{aligned}
& I_{S_{0}}=\text { secondary current at time } t=0 \\
& I_{S_{1}}=\text { secondary current at time } t=t_{1} \\
& I_{S_{2}}=\text { secondary current at time } t=t_{2} \\
& T_{m}=\text { mean lifetime of excited neutral }
\end{aligned}
$$


Then at two different instants of time along the flight path:

$$
\begin{aligned}
& I_{S_{1}}=I_{s_{0}} \exp \left(-t_{1} / T_{m}\right) \\
& I_{s_{2}}=I_{S_{0}} \exp \left(-t_{2} / T_{m}\right)
\end{aligned}
$$

Therefore:

$$
\frac{I_{s_{2}}}{I_{s_{I}}}=\exp \left[-\frac{\left(t_{2}-t_{1}\right)}{T_{m}}\right]
$$

By choosing values of $I_{S_{1}}$ and $I_{s_{2}}$ so that the ratio $I_{S_{2}} / I_{s_{1}}$ is $1 / e$, the value of $\left(t_{2}-t_{1}\right)$ will be equal to $T_{m}$ or the mean lifetime. In each of the cases lllustrated this mean iffetime tums out to be approximately $6.0 \times 10^{-6}$ second.

This lifetime is considerably longer than that of an excited atom which decays by a radiative transition $\left(\sim 10^{-8} \mathrm{sec}\right)$, and the atoms may definitely be considered long lived. However, many authors consider a metastable atom as one in an excited state whose transition to the ground state has a mean lifetime of $10^{-2} \mathrm{sec}$ or Ionger, i.e. by a forbidden transition. The matter is somewhat a case of semantics. It is belleved in the present work that neutral atoms of several energy states are formed in the chargetransfer process. Due to the kinetic energy of the neutrals studied here, the most apparent energy state is that with a half Iife of the order of one microsecond. The neutrals in 
longer-lived excited states are certainly responsible for a portion of the secondary emission, however, the decay of these atoms is not noticeable. Investigations by Kaul ${ }^{4}$ have led to the recent discovery of Iong-lived excited atoms of the noble gases. The lifetimes reported for argon were, for example, $0.6,2.5$, and 20 microseconds. It therefore seems logical that long-lived states with lifetimes in the microsecond range should exist in other elements.

One further remark is appropriate at this point. If the secondary emission by the neutral mercury atoms was exclusively a kinetic energy effect as proposed by Chaudhri and $K_{h a n^{5}}$, there would have been essentially no decrease in the kinetic energy of the neutrals as the target to chargeexchange chamber distance was varied over a range of $40 \mathrm{~cm}$, and consequently no decrease in secondary emission.

${ }^{4}$ W. Kaul, "Die Lebensdauer angergter Reaktionen in Neutralreaktionen, $\mathrm{X}^{*}+\mathrm{Y} \rightarrow \mathrm{XY}^{+}+\mathrm{e}$ ", VI Conference Internationale sur les Phenomena d'Ionization dans les Gaz, 8 au 13 Jullet 1963 a' Paris.

${ }^{5}$ R. M. Chaudhri \& A. W. Kahn, op. cit., p. 526. 
4.3 Quenching of Metastable Atoms by Foreign Gases

Admission of foreign gases into the filight region of the experimental tube had the effect of reducing the secondary emission drastically and even to zero in some instances. The amount of gas admitted proved to be critical. Too little gas had no effect while too much produced ionization of the neutral atoms in the beam. From figure 3.29 it is seen that argon was somewhat more effective in quenching the neutrals. The origin of the peak in the hydrogen curve is uncertain but may be caused as follows. Neutrals in the beam which are not excited may become excited upon collisions with $\mathrm{H}_{2}$ molecules in the pressure range shown. Admission of larger quantities of $\mathrm{H}_{2}$ then tends to quench the metastables.

It was stated in the analysis of the time-of-flight experiment that not all of the excited states had time to decay substantially. With the admission of foreign gases, however, all states have an equal probability of being quenched and thus it is possible for the secondary electron current to reach zero in some cases. The quenching process is essentially an inelastic scattering phenomenon in which the excitation energy of the mercury atoms goes into exciting the $\mathrm{H}_{2}$ or $\mathrm{A}$ atoms. These atoms then drift to the walls of the container where they give up this internal energy by collisions. 
4.4 Secondary Electron Energy

By studying the energy spectrum and energy distribution of the secondary electrons from the target, information may be learned about the incident neutral atom energy. The energy spectra of secondary electrons for neutral atom energies of $300 \mathrm{ev}$ to $1800 \mathrm{ev}$ are plotted in figures 3.210 and 3.211. The characteristic $S$ curves are seen to reach saturation values at high and low values of collector potential. The low saturation value is zero in every case while the high values increase with intake potential. This increase is due primarily to the larger intake of positive ions from the arc. It is partly due to the fact that the kinetic energy of the neutrals plays a small part in the secondary emission. This increase in secondary emission with increasIng intake potential is also apparent in the decay curves of figures 3.25 through 3.28 .

The secondary electron current might be expected to be a step function at the point $V_{4}=0$ rather than the $S$ curve observed. This is not the case for two reasons. For positive values of $V_{4}$ the field between the target and collector essentially penetrates the target surface and lowers its work function. Thus electrons with energies less than the work function can escape from the surface under the 
applied field. The depth of penetration increases with the increasing field strength until a saturation value is reached. For negative collector potentials up to a certain value, some electrons recelve enough energy from the neutrals to overcome the work function and the retarding field.

The value of the potential on the collector which just prevents electrons from leaving the target is a measure of the maximum energy imparted to the metallic electrons by the neutral atoms. From equation 2.23 of chapter 2 the kinetic energy of the electrons leaving the metallic surface is:

$$
\text { (Eq. 2.23) } \quad T=E-\varnothing
$$

Thus if:

$$
\begin{aligned}
& T=e V_{c} \\
& V_{c}=\text { value of } V_{4} \text { which just stops secondaries } \\
& \varnothing=\text { work function of metallic surface }
\end{aligned}
$$

then the energy of the excited neutral becomes $E$ if in the notation of chapter 2 the ground state energy is taken as zero. This argument is only valid for neutral atom energies less than about $800 \mathrm{ev}$. Above $800 \mathrm{ev}$ the kinetic energy of the neutrals begins to play an ever increasing part in the secondary emission as seen by the asymptotic tails of the energy curves of figure 3.211. At neutral energies less 
than $800 \mathrm{ev}$ the secondary electron current falls to zero at $V_{4}=-4.70 \mathrm{v}$ as shown in figure 3.210. Thus the secondary emission is purely a potential effect in this range. Taking $T$ in equation 2.23 as $4.70 \mathrm{ev}$ and the work function $\varnothing$ as $4.25 \mathrm{ev}$ the energy of the exclted neutral atom is found to be $8.95 \mathrm{ev}$ which corresponds to the ${ }^{3} \mathrm{D}_{3}$ state in mercury found by Lichten. ${ }^{6}$ As mentioned previously, neutrals in several energy states are l1kely to exist in the beam. The stopping potential method of measuring energies will only give the most energetic state. Acknowledgment is made of the fact that the target surface was probably mercury coated and the value of the work function can only be approximated. However, the work function for mercury and nickel (target material) are both nearly $4.50 \mathrm{ev}$. The value of $4.25 \mathrm{ev}$ for the target was arrived at by lowering this value by five percent to allow for the fact that a dirty target enhances secondary emission by excited mercury atoms.

The curves of figures 3.212 and 3.213 show the secondary electron distribution for a specific energy spectrum. The energy spectrum here is for a neutral atom kinetic energy of $200 \mathrm{ev}$ which is low enough to ensure that the secondary emission is purely a potential energy effect. Although secondary electron ejection was observed for neutral

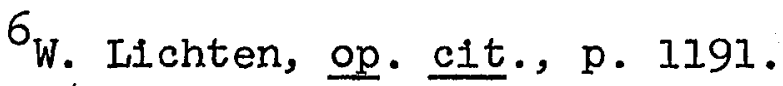


energies as low as $75 \mathrm{ev}$, the energy spectra below $200 \mathrm{ev}$ were not discernible. The electron energy distribution 11lustrated in figure 3.213 is found by differentiation of the energy spectrum curve. This distribution appears to be Maxwellian as was found to be the case by previous workers. However, these early investigators attributed the Maxwellian distribution to the fact that the secondary emission was a kinetic energy effect produced by intense local heating of the target. This theory has been proved incorrect on the basis of the present experiment. The Maxwellian distribution is seen to peak at about $0 \mathrm{ev}$ which indicates that this is the most probable value of the ejected electron energy. This would indicate that the 4.66 and $5.83 \mathrm{ev}$ mercury metastable atoms were responsible for the majority of the secondary emission since their energies are very near the work function of the metal. These atoms would impart just enough energy to the metallic electrons to remove them from the metal with only a fraction of an electron volt of energy left over.

One final point is made with respect to the works of early investigators. In these previous works the energy spectrum curve always had a tall which extended into the region of negative values of $I_{s}$. That is, as the collector potential went negative, so did the target current. This was previously explained as due to neutrals reflected from 
the target striking the collector and ejecting electrons from 1t. These negative currents were in many cases as large as the secondary emission currents from the target itself. It is very unlikely that an excited neutral would strike the target and not revert to the ground state. Thus any reflected neutrals would be in the ground state and unlikely to cause appreciable secondary emission from the collector. This supposition is further refuted by the present work in which a magnetic field, placed perpendicular to the beam of neutrals, completely eliminated the negative target current. It is thus stipulated that these negative currents at the target, observed by previous workers, were actually negative ions and presumably negative $\mathrm{Hg}^{-}$ions. 


\section{CHAPTER 5}

\section{Summary}

By way of summarizing the results of the work presented in foregoing chapters, the following general remarks are in order. Of primary importance is the discovery that excited neutral mercury atoms are formed in charge-transfer collisions between positive mercury ions and mercury vapor atoms. These collisions were found to exhibit resonance characteristics. An excited state having a mean lifetime of approximately 6.0 microseconds was observed. The data Indicated that the $9.0 \mathrm{ev}, 4.66 \mathrm{ev}$ and $5.43 \mathrm{ev}$ metastable states were also present.

The excited atoms were found to have a high capability for producing secondary emission from a metal surface with values of $\gamma$ for the process as high as 0.40 being observed. This secondary emission phenomenon was shown to be due almost entirely to a potential energy transfer between the exclted neutrals and the metallic electrons. While the kinetic energy of the neutrals produced a small secondary electron yield, this effect was seen to be almost negligible. 
During the cours: of the experiment, data were found indicating the presence of negative fons and the evidence was overwhelming in favor of these ions being $\mathrm{Hg}^{-}$. This evidence dictates the need for further study of the plausibility of negative mercury ion formation.

Of great practical interest in regard to the results presented here is the arc-back phenomenon occurring in mercury arc rectifiers. An arc-back occurs when the rectifier suddenly begins to conduct during a negative half cycle thus extinguishing the arc. This may be caused in the following manner. At the instant the a.c. Input voltage to an anode reaches the point of zero magnitude, in a positive half cycle, there are many positive mercury ions distributed in the region between this anode and the cathode. As the anode now becomes negative, the positive ions are accelerated toward It and in the process undergo charge-transfer with the neutral mercury atoms in the region. In the charge-transfer collision, the neutral atom acquires most of the kinetic energy of the positive ion and thus continues toward the negative anode. When the excited neutrals strike the surface of the anode, secondary electrons are released, the necessary energy being provided by the de-excitation of the excited atoms. These electrons are accelerated toward the cathode causing a back current which is greater than normal. If this current reaches a high enough value the rectifier will 
conduct during the negative half cycle and an arc-back will occur. Based on the findings of this research the arc-backs, if produced in the manner described above, could be prevented by introducing optimum quantities of argon gas into the rectifier tube.

It is thus noted that the research undertaken as the topic of this dissertation provides basic scientific knowledge as well as lending itself to a practical application. 


\section{BIBLIOGRAPHY}

\section{$\underline{\text { Books }}$}

Bates, D. R., Atomic and Molecular Processes, New York and Iondon, Academic Press, 1962.

Brown, S. C., Basic Data of Plasma Physics, New York, Wiley and Sons, 1959.

Ioeb, L. B., Fundamental Processes of Electrical Discharges in Gases, New York, Wiley and Sons, 1939.

\section{Periodicals}

Arnot, F. I. \& Milligan, J. C., Proc. Roy. Soc., Vol. Al56 (1936) pp. 359 and 538.

Berry, H. W., Phys. Rev., Vol. 74 (1948), p. 848.

Chaudhri, R. M. \& Kahn, A. W., Proc. Phys. Soc., Vol. 61 $(1948)$, p. 526 .

Cobas, A. \& Lamb, W., Phys. Rev., Vol. 65 (1944), p. 327.

Coulliette, J. H., Phys. Rev., Vol. 32 (1928), p. 636.

Hagstrum, H., Phys. Rev., Vol. 96 (1954), pp. 324 and 336.

Holstein, T., J. Phys. Chem. Vol. 56 (1952), p. 832 .

Lichten, W., Phys. Rev., Vol. 109 (1958), p. 1191.

Massey, H. S. W., \& Smith, R. A., Proc. Roy. Soc., Vol. A155 (1938), p. 472 .

Messenger, H. A., Phys. Rev., Vol. 28 (1926), p. 962.

Oliphant, M. L. E., Proc. Roy. Soc., Vol. Al24 (1929), P. 228 Sena, I., J. Exp. Theoret. Phys. (U.S.S.R.), Vol. 9 (1939), p. $13 \overline{20}$. 
Sloane, R. H. \& Press, R., Proc. Roy. Soc., Vol. Al68 (1938) p. 284 .

Sonkin, S., Phys. Rev., Vol. 43 (1933), p. 788.

Webb, H. W., Phys. Rev., Vol. 24 (1924) p. 113.

\section{Miscellaneous}

W. Kaul, "Die Lebensdauer angergter Reaktionen in Neutralreaktionen, $\mathrm{X}^{*}+\mathrm{Y} \rightarrow \mathrm{XY}^{+}+\mathrm{e}$ ", VI Conference Internationale sur les Phenomena d'Ionization dans les Gaz, 8 au 13 Juillet 1963 a' Paris. 